SSCL --272

DE91 002525

\title{
EARLY INSTRUMENTATION PROJECTS AT THE SSC*
}

\author{
D. J. Martin, L. K. Mestha, S. A. Miller, and R. Talman \\ Superconducting Super Collider Laboratory ${ }^{\dagger}$ \\ Accelerator Division \\ 2550 Beckleymeade Avenue \\ Dallas, Texas 75237
}

April 1990

\section{DISCLAIMER}

This report was prepared as an account of work sponsored by an agency of the United States This report was preir Government. Neither the United States Governmed, or assumes any legal liability or responsiemployees, makes any warmesters, or usefulness of any information, apparatus, product, or bility for the accuracy, completeness, or usefulness of any inforinge privately owned rights. Referprocess disclosed, or represents that its use would no in, or service by trade name, trademark, ence herein to any specific commercial product, process, or service by its endorsement, recommanufacturer, or otherwise does not necessarily constitute or mply its endereof. The views

- $\because$ mendation, or favoring by the United States do not necessarily state or reflect those of the

and opinions of authors expressed herein do not
United States Government or any agency thereof.

\footnotetext{
*Presented at the International Industrial Symposium on the Super Collider, Miami Beach, Florida, Marcn 14-16, 1990.

†Operated by the Universities Research Association, Inc., for the U.S. Department of Energy under Contract No. DE-AC02-89ER40486. 


\title{
EARLY INSTRUMENTATION PROJECTS AT THE SSC
}

\author{
D. J. Martin, L. K. Mestha, S. A. Miller and R. Talman
}

Superconducting Super Collider Laboratory

2250 Beckleymeade-MS 1046

Dallas, TX 75237

\begin{abstract}
Conceptual designs for some SSC instrumentation is given. Stripline beam position monitors, appropriate for cryogenic operation are described, along with plans for their $A / D$ conversion and recording. A global timing system based on fibre optics is described; it is to be capable of \pm 100 psec accuracy over many tens of kilometers. Stabilization is pattemed after a scheme in ust at CERN. Timing pulse's (roughly $60 \mathrm{MHz}$ ) as well as pulses synchronized to the various frequency modulated RF systems are distributed and scaled by digital clocks situated at those locations where accurate timing is required. Finally, a digital control circuit to be used for synchronizing beam transfer from the Low Energy Booster to the Medium Energy Booster is described. It is based on controlling the relative phases of the two RF systems even though one of the frequencies is variable.
\end{abstract}

\section{INTRODUCTION}

In this paper three related instrumentation projects at the SSC are described. These projects are still at the conceptual design level, with acquisition and prototype development either just beginning or not yet started. The first report describes beam position measurement. The second describes a distributed timing system capable of providing timing signals with accuracy \pm 0.1 nsec anywhere on the site. The third report describes the Low Energy Booster (LEB) to Medium Energy Booster (MEB) beam transfer synchronizer.

\footnotetext{
*Operated by the Universities Research Association, Inc., for the U.S. Department of Energy under Contraci No. DE-AC02-89ER40486.
} 


\section{SSCL BEAM POSITION MONITORING SYSTEM}

The BPM system contains the most important instrumentation for beam control. Position pick-ups, each with four ciectrodes, will be located at most quadrupoles in all the accelerators. Doublet pulses of 1 ns duration and 16 ns separation are produced as the beam traverses the pickups. The peak-to-peak amplitude of pulses varies from $0.5 \mathrm{~V}$ at machine commissioning to $20 \mathrm{~V}$ at operating intensity. The detector signals will be processed and digitized in the niches, and the digitized data stored in registers for readout by the control system. At least three methods of analog signal processing are being considered to meet accelerator requirements.

Each sensing device is a detector consisting of four $15-\mathrm{cm}, 50$ ohm strip transmission lines placed above, below, and to both sides of the beam as shown in Figure 1 and Figure 2. To maximize the signal to noise ratio, each electrode subtends most of one quadrant. So that the electrodes will not be aperture defining elements, the beam tube bulges out around them. The electrodes are also recessed $2 \mathrm{~mm}$ outside the aperture so that synchrotron radiation cannot strike them. The characteristic impedance of the four electrodes must be matched within $\pm 0.25 \%$ to hold the electrical to mechanical center difference to 0.005 in., and to be within $\pm 0.5 \%$ of 50 ohms to control reflections at the cable interface. The detectors are rigidly welded to the spool pieces in each half-cell for alignment purposes and therefore operate at $4 \mathrm{~K}$. The 8000 vacuum feedthroughs used in the collider must isolate beam vacuum from liquid helium. Because of their great quantity and inherent difficulty of replacement they must be very reliable (MTBF $>870 \times 10^{6} \mathrm{hrs}$ ). The feedthrough and cable to the outside of the cryostat form an integral assembly and are composed of 316 Stainless Steel (S.S.) and $\mathrm{Al}_{2} \mathrm{O}_{3}$ ceramic. The cable dielectric is $\mathrm{SiO}_{2}$. The 0.142 in. dia. S.S. jackets of the four cables form a part of the hermetically sealed cryostat which confines the liquid helium. The cables will hold off $20 \mathrm{~atm}$. LHe, be radiation resistant, tolerate welding and be very rugged.

Since beam detector directionality is not required in the collider, one end of each stripline is shorted. This measure saves construction cost, reduces heat leak, and improves reliability. The integral cable assemblies mate to SMA bulkhead vacuum feedthroughs which isolate guard vacuum from atmosphere. These feedthroughs are at the outside surface of the cryostat and are readily replaceable if damaged. All BPM signals will be brought to processor racks in the tunnel niches from the adjacent 3 consecutive upstream and downstream halfcells. The longest cable runs are $270 \mathrm{~m}$.

The BPM electronics must provide position and intensity signals under various operating conditions: during machine commissioning; at full intensity collider operation; in tault diagnosis; and during specialized accelerator studies. Optimal performance in a variety of applications requires front-end processing tailored to the various modes and ring locations.

For maximum sensitivity and maximum dynamic range in sensing trains of bunches separated by $5 \mathrm{~m}$ intervals, down-converted amplitude-to-phase conversion is used 1 . Signal processing is done using only one harmonic of the RF bunching frequency. With $15 \mathrm{kHz}$. wide bandpass filters in the IF section and $15 \mathrm{~dB}$ noise figure limiters, resolution of $100 \mathrm{~nm}$ should be achieved. The good sensitivity of these channels at low beam current will be valuable in steering the beam through the first turn commissioning. For this purpose, it is not necessary to instrument every half-cell, and for that reason, AM/PM processing will be used at the BPM stations adjacent to the niches, every three cells. These locations use $30 \mathrm{~m}$ of $7 / 8$ 


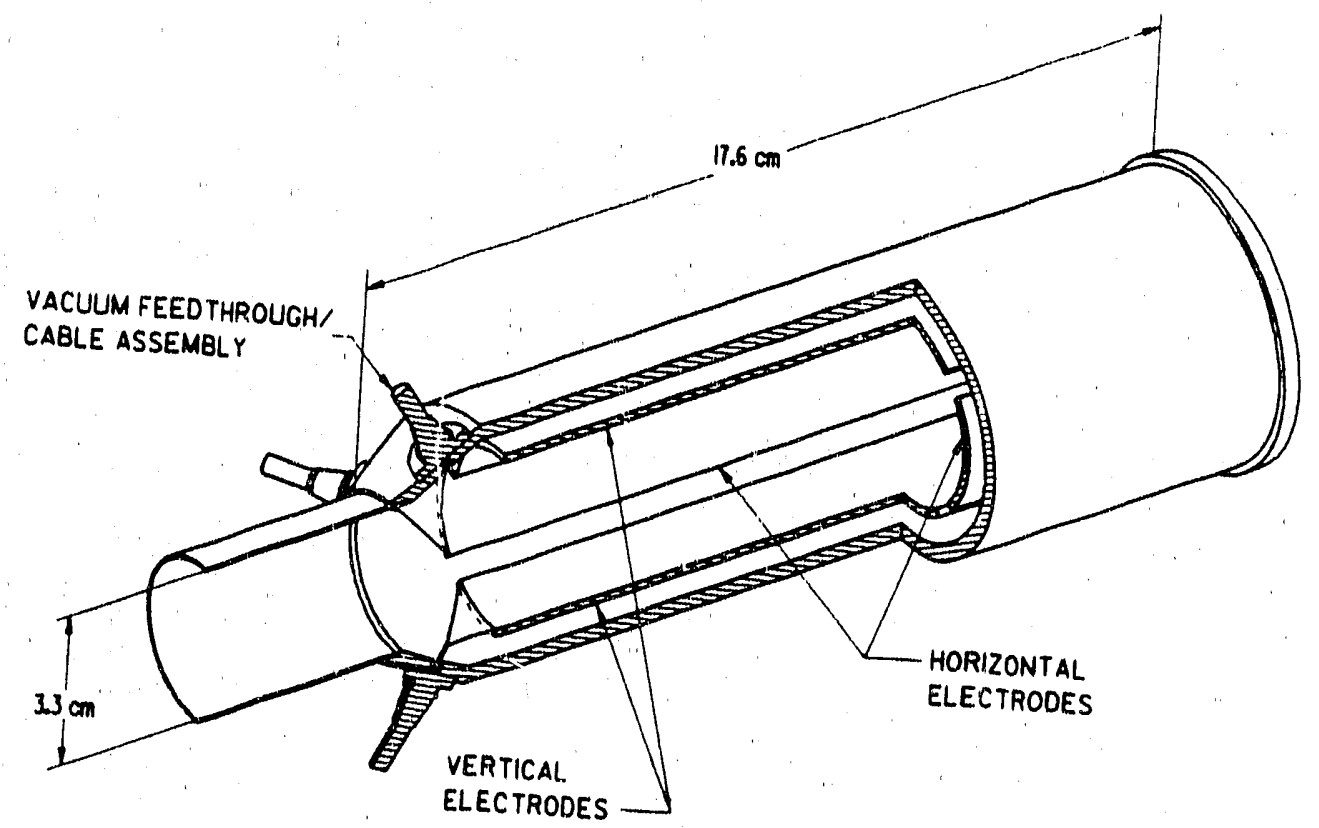

Figure 1. Isometric vie's of beam position monitor.

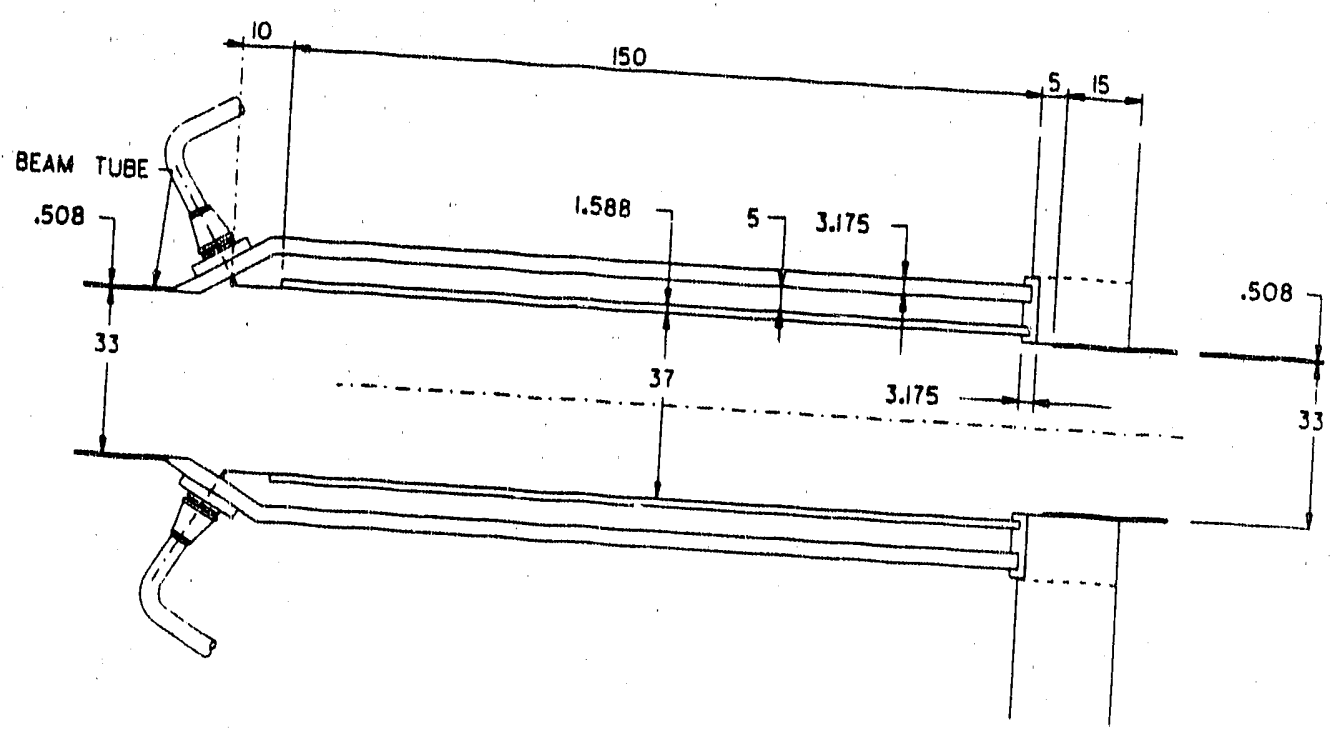

NOTE:

ALL DIMENSIONS ARE IN MILLIMETERS

Figure 2. Plan view of beam position monitor. 
in. solid copper jacketed corrugated cable, making the niche BPM's the most sensitive locations in the accelerator. Position monitoring near the IR's will also use this system.

At the BPM's most distant from the niches, skin effect losses even in high quality, low loss cable remain excessive for the pulse risetime to be preserved. Therefore, located close to the pickups, will be peak detection circuits which effectively down-convert the high frequency components. The diode detectors provide the bulk of the front-end BPM processing, are the least costly of the methods, and use lower quality RG-213 cable to transmit rectified pulses to the niches. The diode circuits have limited dynamic range, about $35 \mathrm{~dB}$, and must be hardened against radiation. To measure the transverse position of a particular bunch, adjustable timing signals are used to gate the 12-bit ADC. These timing signals are derived from the global timing system described in the next section. Each station will be equipped with First In First Out (FIFO) memories that retain the most recent ten thousand turns of data. These will be used for occasional readout, for example for post mortem after a beam abort to reconstruct the beam orbit. Under special conditions, all position monitors must be able to measure orbit distortions of about 10 microns peak amplitude to locate orbit cusps.

For specialized beam studies, bunch-by-bunch and turn-by-turn processing will be implemented in a few places in each accelerator. Such a system will be able to measure the position of any bunch, any sequence of bunches, the same bunch on every beam orbit (turn), or any other definable bunch pattern. The electronics will employ 8-bit $100 \mathrm{MHz}$ flash $\mathrm{AD}$ converters, digital intensity normalizing, and FIFO memories to record position histories. To obtain reasonable resolution, wideband sum and difference signals are obtained using hybrid transformers integral to each detector. The flash converters clocked at $60 \mathrm{MHz}$ take one sample of the vertical position, horizontal position, and intensity of each bunch. The ratios of differences to sums are taken digitally and are written into fast memories, which can be read by the control system. A $100 \mathrm{Kbyte}$ memory could store the position data of 6 full turns, or the position of a single bunch on $10^{5}$ turns. The electronics would be similar to the front-end processing done in the Tevatron beam dampers?

\section{SSCL PRECISION TIMING SYSTEMS}

\section{Introduction}

The timing systems of the SSCL generate the individual tining triggers in the accelerator complex. They are used by the synchronization of beam transfer, beam dump and beam position data acquisition systems, which require a jitter of $\leq 100$ psec. Their use by the ramping and corrector magnets require a jitter of $\leq 1 \mathrm{msec}$. Finally, the systems provide a phase compensated RF reference. It is no small task to distribute a timing signal with a jitter and precision of $\leq 100$ psec over a geographic area of approximately 200 square miles. The collider itself will contain 161 niches, spaced evenly 540 meters apart. Each of these niches must receive the high precision timing signal.

The precision timing system incorporates features from the Tevatron, CERN and the SLC project at SLAC. An SSCL timing system consists of a distribution network which carries $R F$ reference signals and timing modules which count the number of cycles of the RF reference signal. At predetermined counts, timing modules produce trigger signals. For more precise timing, individual RF cycles can be subdivided. 
There are 7 different timing systems: the Linac, LEB, MEB, HEB, SSC Top, and SSC Bottom Beam Syncs, and Global Timing. The Beam Sync systems are distributed to their respective accelerators, while the Global Timing is distributed to all accelerators. The systems perform the same functions but have different master clcoks. The master clock of a system is the origin of the RF reference signal of a system. For the Global Timing, the master clock is phaselocked to the $1,000,000$ th harmonic $60 \mathrm{~Hz}$ line frequency, or $60 \mathrm{MHz}$. For the Beam Syncs, the master clock is phaselocked to the RF in the accelerator cavities.

During acceleration, the Beam Sync systems are approximately $60 \mathrm{MHz}$, hut do sweep a significant frequency range ( 47 to $60 \mathrm{MHz}$ for the LEB). Other than the effects of the FM signal, the systems may be considered identical in function and construction. We will confine our observations to the Global Timing System (GTS).

\section{Goals of the GTS}

We require that timing modules trigger simultaneously to \pm 100 psec anywhere in the accelerator complex. Jitter and repeatability of individual modules should be better than 100 psec. A high mean time between replacement (MTBR) is required, as the GTS must be operating properly for the collider to operate. MTBR should exceed 100,000 hours for the individual modules. Self diagnostics should allow faults to be determined during production and as an aid to troubleshooting during installation and operation. If possible, the system should give indications of how soon various failures are predieted for modules.

\section{Network Topology}

The precision, cost and reliability of the timing system is strongly influenced by how the signals are actually routed - the distribution network topology. The intention of the distribution network topology is to minimize jitter, uncertainty, drift and cost, while maximizing reliability.

The simplest distribution network, a horseshoe (Figure 3a) would have one fiber strung from niche to niche, with a repeater at each niche. This would minimize cost of fiber used to $\$ 262,500$, but would result in a probable increase in jitter, uncertainty and drift of 22 times that of a single repeater, and a worst case increase of 484 times that of a single repeater. The loss of a single repeater would cripple all the down stream timing circuits, or as much as $1 / 2$ of the collider.

The opposite extreme, as shown in Figure $3 b$ ( star net), would be to string a separate fiber to each niche, which would require bundles of as many as 484 fibers to be strung in the cable conduit. It would minimize the jitter, uncertainty and drift to that of one repeater, but would require $\$ 63,381,000$ of fiber. Loss of any repeater would affect only one niche.

A compromise star-star network (Figure 3c) would distribute one fiber to each sector, which would then subdistribute to each niche. This results in bundles of no more than 13 fibers. The total cost of fiber is $\$ 982,200$. The probable jitter, uncertainty and drift are 1.41 times that of a single repeater, and the worst case is 2 times that of a single repeater. Loss of a subdistribution repeater would affect only one niche, while loss of a distribution repeater would affect at most one sector. The star-star network will require 10 more repeaters than the other systems. The system is shown in more detail in Figure 4. 

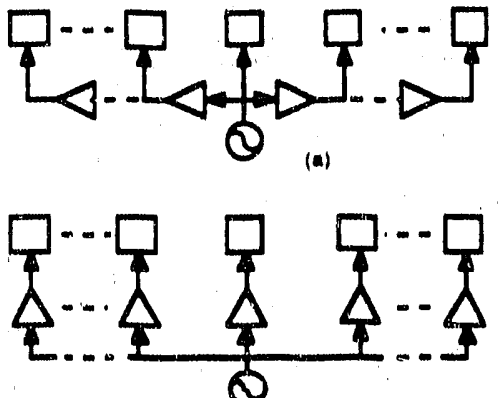

(b)

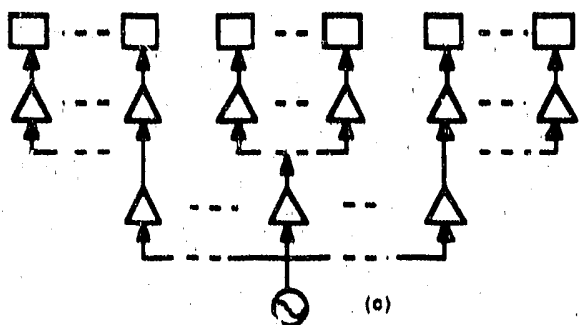

FIG. 3: TIMING DISTRIBUTION NETWORK

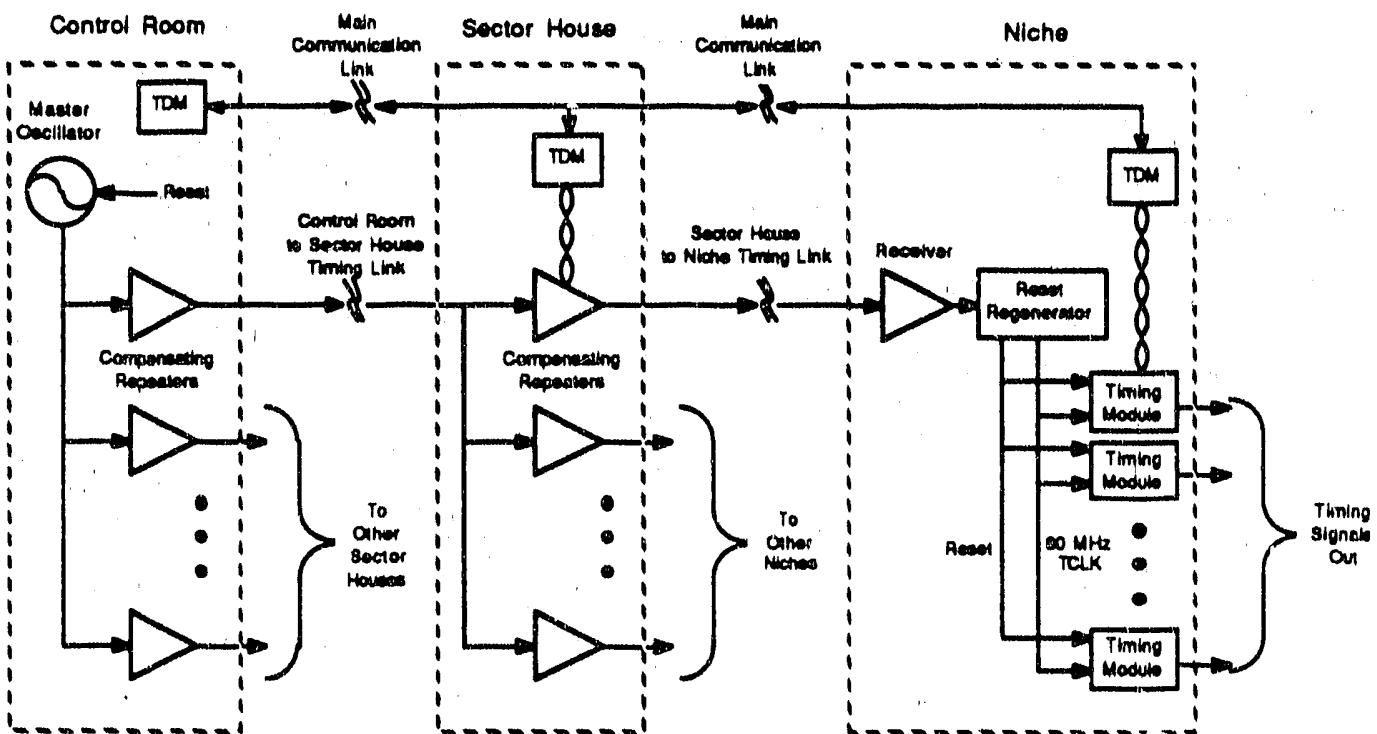

FIg. 4: TIMING SYSTEM BLOCK DIAGRAM

Installation of the network is no small task. There is some question of the long term radiation resistance of the fiber, as silica becomes more opaque as it is exposed to neutrons. The fibers are shielded underneath a minimum of 18 inches of rubble and concrete. The niches are 540 meters apart, and contain the pull boxes for the fiber. To reduce the number of 
splices required, the tiber bundle is pulled from one niche and respooled at the next. This process is repeated, with appropriate breakouts being made, until the entire network is strung.

\section{High Accuracy Timing Signal Transmission}

Either coaxial or fiber optic cables could be used to distribute the timing signal. Fiber optics are superior to coax in their cost, attenuation, variation of propagation velocity with temperature and common mode noise rejection characteristics. The radiation resistance of fiber optics is comparable to coax ${ }^{3}$. Fiber optic links have also been used in the Tevatron and LEP timing systems.

Variations in temperature of $\pm 5^{\circ} \mathrm{C}$ will make variations of as rnuch as $\pm 13 \mathrm{nsec}$ in triggering time from one side of the collider to the other due to the variation in propagation velocity with temperature. A technique originally developed by Peschardt and Sladen ${ }^{4}$ and enhanced for this application allows the temperature variation to be compensated (Figure 5). It does this by measuring the phase shift through the fiber round trip and compensating for any variation.

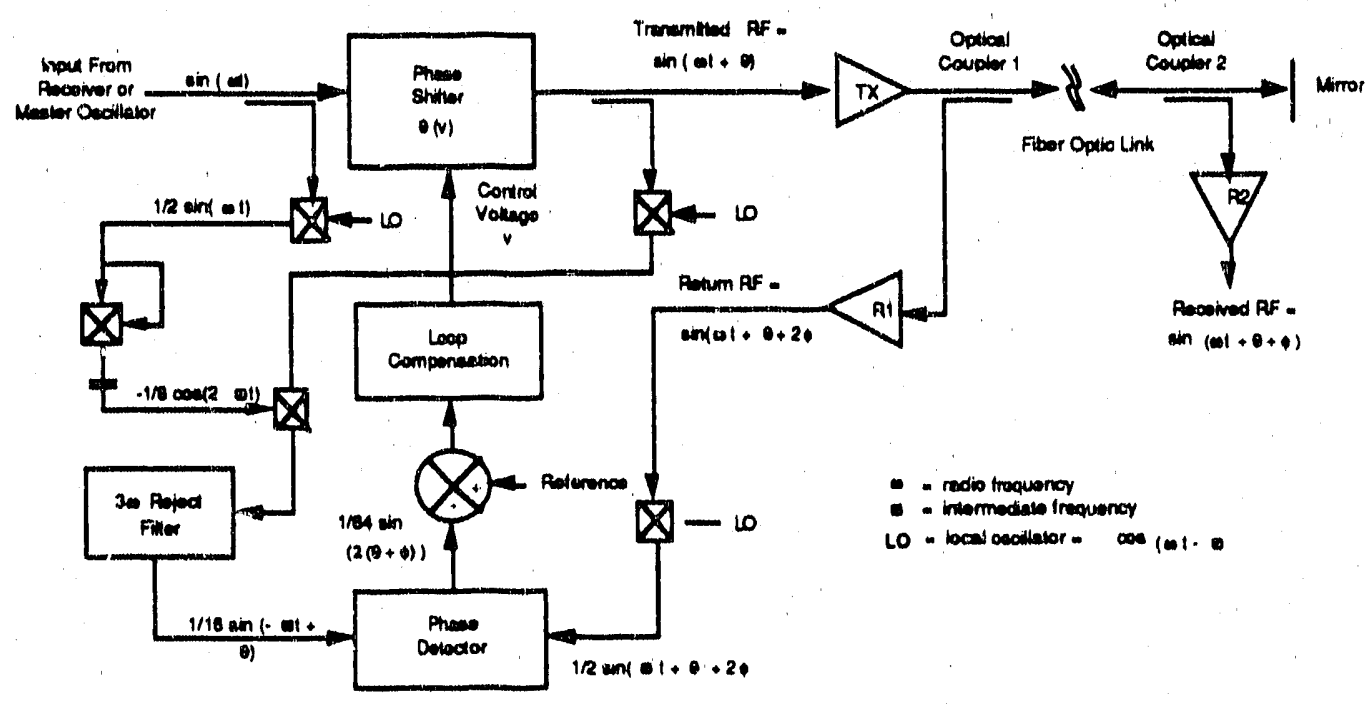

Fig. 5: PHASE-COMPENSATED FIBER OPTIC LINK (Adapted from Poechardt and Sladon)

As seen in Fig. 5, an input signal, designated "From Receiver/Signal Processor", is assumed to be the phase reference $V_{i}$

$$
V_{i}(t)=\sin (\omega t)
$$

This is passed through the phase shifter, and results in $V_{1}$

$$
V_{t}(t)=\sin (\omega t+\theta(v))
$$


where the phase shift $\theta$ is a function of the control voltage $v_{,} V_{\text {, is }}$ is then transmitted over a single mode optical fiber. The media adds an additional phase shift of $\phi=\omega \tau$, where $\tau$ is the one way time delay of the media. Optical Coupler 2 picks off a fraction of the signal, which is converted into $V_{\text {rec }}$ by Receiver $R X 2$.

$$
V_{\text {rec }}(t)=\sin (\omega t+\theta(v)+\phi)
$$

The signal which is not absorbed by RX2 is reflected back to the transmitter, where it is picked of by Optical Coupler 1 and converted into $V_{\text {ret. }}$

$$
V_{\text {ret }}(t)=\sin (\omega t+\theta(v)+2 \phi)
$$

$V_{i}$ is down converted by a local oscillator LO. The down converted frequency is represented by $\Phi$. The frequency of LO is set so $\Phi$ is about $100 \mathrm{KHz}$.

$$
\begin{gathered}
V_{L O}(t)=\cos ((\omega-\varpi) t) \\
\left.V_{i}(t) V_{L O}(t)\right|_{\text {LowPass }}=\left.\sin (\omega t) \cos ((\omega-\varpi) t)\right|_{\text {LowPass }}=\frac{1}{2} \sin (\varpi t)
\end{gathered}
$$

This down converted reforence is then multiplied by itself and the DC component removed, resulting in $-\frac{1}{8} \cos (2 \varpi t)$. This is multiplied by a down converted image of $V_{t}$ and the 3rd harmonic removed:

$$
-\left.\frac{1}{8} \cos (2 \omega t) \sin (\varpi t+\theta(v))\right|_{\varpi \text { BandPass }}=\frac{1}{16} \sin (-\varpi t+\theta(v))
$$

$V_{\text {ret }}$ is also down converted, resulting in

$$
\begin{aligned}
\left.V_{\text {ret }}(t) V_{\text {LO }}(t)\right|_{\text {LowPass }} & =\left.\sin (\omega t+\theta(v)+2 \phi) \cos ((\omega-\sigma) t)\right|_{\text {LowPass }} \\
& =\frac{1}{2} \sin (\varpi t+\theta(v)+2 \phi)
\end{aligned}
$$

When these last two signals are multiplied together in the phase detector and harmonics removed, the result is $\mathrm{V}_{\mathrm{PD}}$ :

$$
\left.V_{P D}(t)=\frac{1}{64} \cos (2 \theta(v)+2 \phi)\right)
$$

If the error amplifier and the loop filter feedback to the phase shifter, they will cause

$$
V_{P D}(t)=\frac{1}{64} \cos (2(\theta(v)+\phi))=\text { constant }=V_{\text {ref }}
$$

or

$$
\theta(v)+\phi=\text { constant }
$$


provided that $\theta(v)$ is approximately linear and has a positive slope, and the loop filter stabilizes the loop. But this last equation is merely the condition that the phase shift from the input to the receiver output be con tant. Therefore any changes in $\phi$ with time, temperature, etc, will result in a change in $\theta(v)$ which cancels it out.

At CERN, this resulted in a reduction of the variation of the delay of the LEP RF reference line, the longest of whicil is $7.9 \mathrm{Km}$, from $\pm 8 \mathrm{nsec}$ with the feedback loop off to $\pm 19 \mathrm{psec}$ with the feedback loop on, over a $20^{\circ} \mathrm{C}$ temperature range. Extrapolation to the requirements of the SSC show that \pm 100 pseo stability should be achievable.

Two additional modifications are required for the SSC. The system must be modified when used as a Beam Sync for the lower energy boosters. This is because of the wide variation in operation frequency of these accelerators. Since the phase compensated line keeps the total phase shift through a line constant, the net time delay of the system is a function of frequency:

$$
\tau=(\theta(v)+\phi) / \omega=\text { constant } / \omega
$$

This may be handled by using a TDC which keeps track of the total transit time. This number is then used to control a variable delay line, rather than a variable phase shifter. Under this control scheme, the system keeps the time delay, rather than the phase, constant (Figure 6). An alternative may be to account for the time delay variation with frequency in the database of the central control computer, and redistribute new trigger times as required. The hardware solution is perhaps better, since it requires about the same level of complexity and would greatly reduce the amount of communication required between the timing modules and the central computer.

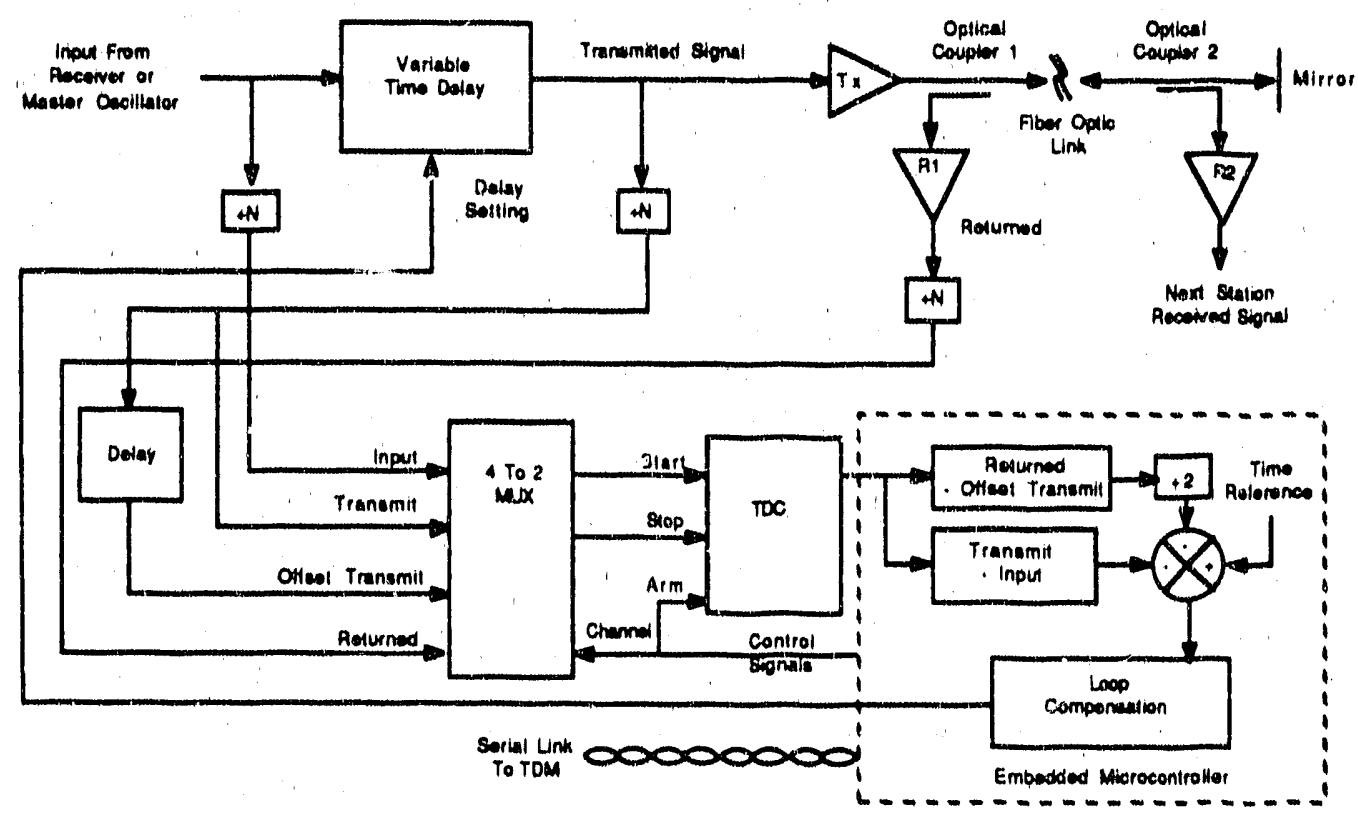

FIg. 6: TIME-COMPENSATED FIBER OPTIC LINK 
A reset mechanism must also be provided which allows the timing modules to be set back to a zero count (Figure 4). This is done by removing a pulse from the transmitted pulse train. The missing pulse is detected and generates a reset signal. The reset signal is used by timing modules to reset their internal counters to zero. The RF timing frequency itself is regenerated using a phase locked loop (PLL) in a process similar to that used in high speed serial transmissions (Figure 7). This regeneration process greatly enhances the phase stability and noise margin of the signal. The signal is then distributed locally to the niche or recombined with the reset signal for subdistribution.

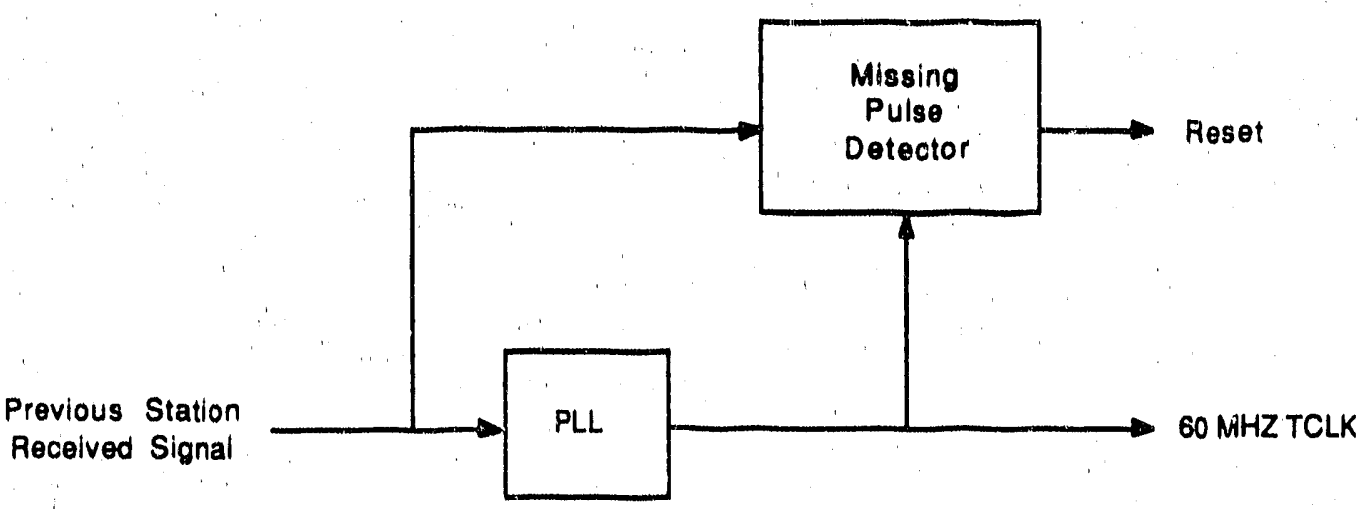

Fig. 7: RESET REGENERATOR

A diagnostic signal is returned from the loop filter to the central computer. This diagnostic measures the required compensation and hence is a direct measurement of the phase shift of the line. Each repeater's nominal phase shift can be recr rded in a database in the central computer and periodically compared with the current value. A large shift in compensation would indicate that some component it the system is shifting drastically and will need to be replaced at the next maintenance cycle.

\section{Timing Module Operation}

The timing modules themselves are elementary (See Figure 8). The number of requested time clock cycles required is down-loaded from the central computer to an individual timing module via a dedicated serial link to a Time Domain Multiplexer/Demultiplexer. This is received and stored by a dedicated microcontroller unit (MCU). The MCU holds the least significant bits of the requested time word (RTW) on the input of an equality detector.

The RF reference signal TCLK enters the High Speed Counter, which simply tallies the number of clock cycles since the counter was last reset, or since overflow. The size of the High Speed Counter is considerably smaller than the RTW, but gives the MCU enough time to anticipate the instant when the R:W is equal to the total number of cycles since reset . When the number of cycles counted by the High Speed Counter is equal to the least significant bits of the RTW, a pulse is generated which alers the High Speed Arming Circuit, which in turn alerts the MCU. The MCU then compares the rest of the RTW with the number 


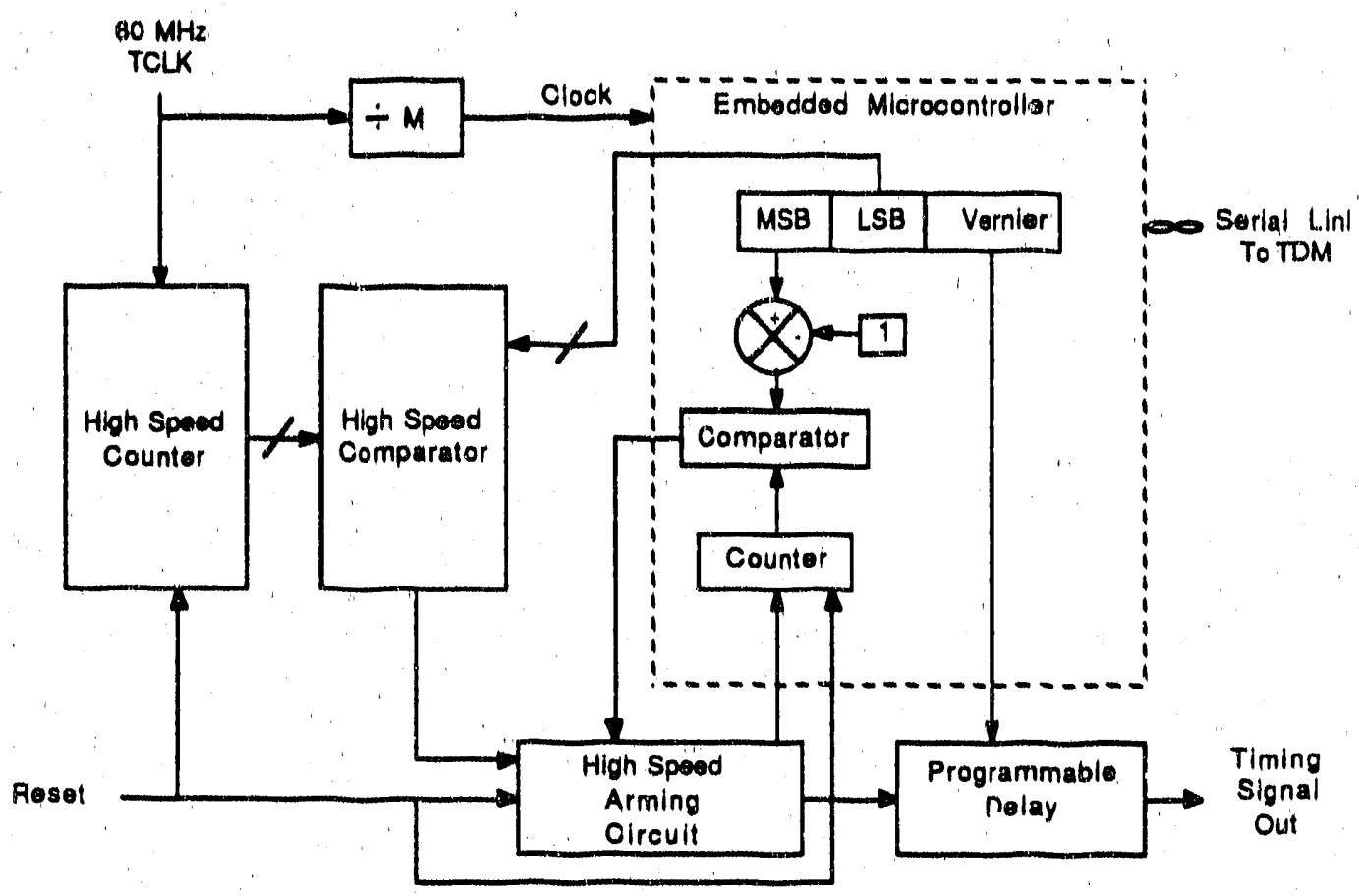

FIg. 8: TIMING MODULE

of alerts received from the High Speed Arming Circuit. Once the rest of the RTW minus one matches with number of alerts, the High Speed Arming circuit is armed by the MCU. When the next equality is detected, the High Speed Arming Circuit produces an output pulse. This pulse is delayed by a fraction of a cycle by a programmable delay line, which acts as a vernier on the RTW.

The MCU clock is slaved to TCLK, assuring that its software counters are synchronized to TCLK at least to the accuracy required to properly anticipate and arm the High Speed Arming circuit. More than one timing module can be serviced by one MCU, which allows a reduction in cost and complexity, especially in the serial communication link to the central computer. For timing applications which do not require very high accuracy, the vernier delay line can be left out. For applications which require only crude timing, most of the high speed circuitry may be omitted.

\section{Timing System Calibration}

The central computer provid:s RTW's which are compensated for delay time from niche to niche, FM effects in the lower energy boosters, etc. Each of the timing systems must be calibrated and the calibration stored in the central database. For example, the difference in phase between two clocks separated by a communication link of time delay $\tau$ is given by 


$$
\begin{aligned}
\Delta \phi & =\int_{t_{0}}^{t_{0}+\tau} \omega(t) d t \\
& \equiv \tau \omega\left(t_{0}\right)
\end{aligned}
$$

It is clear that the time delay from the master oscillator to each niche must be known with high accuracy. This calibration procedure is done in two steps. The first, a crude calibration, allows the SSCL to be commissioned but does not provide the ultimate level of accuracy of the system to be achieved. The technique is simply to replace the master oscillator with a high stability atomic clock, and then to synchronize : second atomic clock to the temporary master. This atomic clock is portable and connected to a timing module. Once this is done, the second atomic cluck and its attendant timing module may be moved quickly from niche to niche and the mobile timing module compared with the count achieved by the the timing; modules at each niche.

The accuracy of this system is limited by the maximum speed with which a precision atomic clock mis be moved from point to point in the system and by the phase stability of state-of-the-art atomic clocks. The best accuracy requires a hydrogen maser clock. The speed of a clock through the collider is limited to about $15 \mathrm{Km} / \mathrm{hour}$. An overland route might speed this up considerably, but might unduly vibi ute the atomic clock.

The second calibrtion step can proceed once protons can circulate through the collider. The protons travel at a known velocity, for a known distance, and their time of flight from niche to niche can be determined with arbitrary accuracy und precision. By using the timing modules to nots the exact time of arrival of a s gle bunch of protons, the time delays of the system may be culibrated out to an accuracy of better than $100 \mathrm{psiec}$ at all locations in the accelerator complex.

\section{SSCI, BEAM TRANSFER SYNCHRONIZATION}

\section{Introduction}

At present there are two schemes for achieving synchronous transfer of bunched beams from one circular accelerator to another in various laboratories around the world 5.6 . In the Booster to Main Ring transfer at Fermilab, a phase-locking scheme is in use. In this approach, at a suitable time before injection to the Main ring, the phase and frequencies of the Booster RF are locked to the Main Ring RF system. When the RF phase error is zero synchronous transfer is triggered. In the corresponding transfer at KEK in Japan the phase matching is done by using a phase-slippage scheme. In this scheme, at a predetermined time before the transfer, the Booster RF frequency is offset by $10 \mathrm{Khz}$ relative to the Main Ring RF frequency. As a result, since the Main Ring RF frequency is constant, the RF phase of the Booster ring slips relative to the Mair Ring. Phase coincidence is detected and then a kicker system is fired to transfer the /xeam. Some emittance dilution occurs owing to the mismatch of bunch spacing; that is, an inherent drawback of the system.

The phase-locking scheme gives good results when there is sufficient time, first to achieve the RF lock, and then to perform the "cogging" operation in which the bunch pattem is rotased into its correct orientation. In the SSC, especially for the bearn transfer between the 
Low Energy Booster (LEB) and the Medium Energy Booster (MEB), this scheme is not suitable because at top energy the LEB is ope ating very close to transition which makes the cogging time too large 7,8 and the peak B-field does not have a long flat top since it is derived fiom the biased sinusoidal resonant power source. (It has been judged impractical to derive a long flat top by switching an auxiliary power supply). However, for all other transfers at SSC this scheme can be applied since the B-field can be held constant until the transfer process is completed.

The ideal scheme for synchronizing beam transfer from the LEB to MEB is to keep control of the relative beam phase throughout the acceleration. Since the frequency or the LEB is modulated by about $20 \%$ during acceleration, the concept of relative phase must be generalized and for that purpose we introduce a new term "synchroni-ging phase" fn: phase that is controlled through the cycle and acquires its stan'prd meaning when the two frequencies become equal. In this methou, transfer is riggered at the instant the synchronizing phase vanishes. This avoids emittance dilution due to mismatch of bunch spacing as in the phase-slippage scheme, even with large bunch separation (e.g. $90 \mathrm{~m}$ instead of the nominal 5m). The scheme has twr essential elements. First, it involves the accurate detection of the synchronizing phase error by comparing the measured values with the known "trip plan" stored in the computer. The trip plan and the synchronizing phase are related terms with the trip plan being lueal values of the synchronizing priase at discrete intervals through the cycle. Second, the measured phase error is passed through a feedback controlier digitally, so that a compensating RF frequency shift is generated to minimize the error. In this way the synchronizing phase is controlled to a pre-programmed trajectory. In the following section we discuss the method qualitatively and show how it will be possible to detect the synchronizing phase at regular intervals. The feedback controller is not explained. A detailed analysis of the scheme is contained in reference ${ }^{9}$.

\section{Principle of the Phase-Control Scheme}

To begin with let us assume that there is no frequency error in the LEB and MEB RF systems. Transfer line delays and measurement errors in beam position (due for example, to coupled bunch oscillations) are ignored. Also, to understand the basic principle, let us assume that the synchronous phase of the reference particle in the beam bunch is constant throughout the acceleration cycle. With these assumptions in mind we can write an expression for the path length covered by the beam for a ti ne duration of $\tau$ as follows.

$$
L_{L E B}(\tau)=\frac{2 \pi R_{i}}{h_{L}} \int_{0}^{\tau} f_{L}(t) d t+L_{L E B}(0)
$$

$$
\text { where, } \begin{aligned}
\tau & =\text { Time interval } \\
R_{L} & =\text { Radius of the orbit } \\
h_{L} & =\text { Harmonic number for the LEB } \\
f_{L}(t) & =R F \text { frequency of the LEB }
\end{aligned}
$$

(Numerically $L_{L E B}$ is roughly $10^{7} \mathrm{~m}$ at transfer time. Since a longitudinal uncertainty of less than $10-2 \mathrm{~m}$ is required at that time, it is clear that absolute control of all parameters to the corresponding precision of one part in $10^{9}$ is an impract: al way of synchronizing transfer). 
Taking advantage of the constancy of the MEB frequency, the path length in the MEB can be written:

$$
\mathrm{L}_{\mathrm{MEB}}(\tau)=\frac{2 \pi \mathrm{R}_{\mathrm{M}}}{\mathrm{h}_{\mathrm{M}}} \mathrm{f}_{\mathrm{M}} \tau+\mathrm{L}_{\mathrm{MEB}}(0)
$$

The difference in path lengths, $L_{\psi}=L_{M E B}-L_{L E B}$, is equal to the synchronizing phase. The phase when defined in this form has units of length. To solve for $\mathrm{L}_{\psi}$, the following equation is used for the LEB RF frequency with usual notations.

$$
f_{,}(t)=\frac{h_{L} c}{2 \pi R_{L} \sqrt{1+\left(\frac{M_{p} c^{2} / e}{\rho c B(t)}\right)^{2}}}
$$

Where $\rho$ is equal to the effective bending radius. The accelerator guide field $B(t)$ varies with time according to the following expression:

$$
B(t)=B_{\min }+\frac{B_{\max }-B_{\min }}{2}(1-\cos \omega t)
$$

Using Eq. 15 in Eq. 13 the path length, $\mathrm{L}_{\mathrm{LEB}}$, is calculated numerically with a ne time step of $1 \mathrm{~ns}$. We assumed $L_{L E B}(0)=0=L_{\text {MEB }}(0)$, i.e., the beam bunch in the $L E B$ was assumed tc have started at the same time as the MEB beam bunch from the reference points. The referesice points were chosen at the beam pick up points nearest to the beginning and the end of the transfer line. For synchronous transfer we would like the synchronizing phase to be equal to zero. Since we are dealing with a circular machine, when the MEB beam bunch has completed one single turn, the LEB beam bunch will have completed a few whole turns plus a semi-turn. The semi-turn is due to the difference in the two RF frequencies throughout acceleration and the fact that the circumference ratio between the MEB and the LEB is not a whole integer. Hence $\mathrm{L} \psi$ can be rewritten in the following form.

$$
L_{\nabla}=L_{\text {MEB }}-L_{\text {LEB }}=\left(N_{\text {MEB }}+\gamma_{M}\right) 2 \pi R_{M}-\left(N_{L E B}+\gamma_{L}\right) 2 \pi R_{L}
$$

Where $N_{M E B}, \gamma_{M}$ and $N_{M E B}, \gamma_{L}$, represents the whole and the semi-rurn completed by the MEB and the LEB reference beam bunch respectively. The whole tum is of no significance for synchronous transfer for obvious reasons. Hence we simply drop it and consider only the fractional part representing the incomplete turm.

For synchronization, at the time of transfer the fractional part $\gamma_{M}$ and $\gamma_{L}$, must be equal to zero. In Figure 9, $L_{\psi}$ is plotted each time the MEB beam bunch returns to the reference point (i,e, when $\gamma_{M}$ becomes zero). It can be seen clearly frorn this figure that after about $47 \mathrm{~ms}$ the LEB beam bunch tends to come back to a fixed point in the orbit more frequently than before. We see three curves that are approaching constancy. (Three curves are due to the fraction " $1 / 3$ " in the MEB to LEB circumference ratio: when the ratio is a whole integer, then we would have only one curve.) The decay is due to the fact that the difference between the two $\mathrm{RF}$ frequencies is narrowing as the time approaches the nominal $50 \mathrm{msec}$ transfer time at which $\mathrm{P}, t)$ is maximum. These curves settle down eventually to a fixed value; for example $68.38 \mathrm{~m}$ is the final settling value for curve 2 . The final settling value can be 


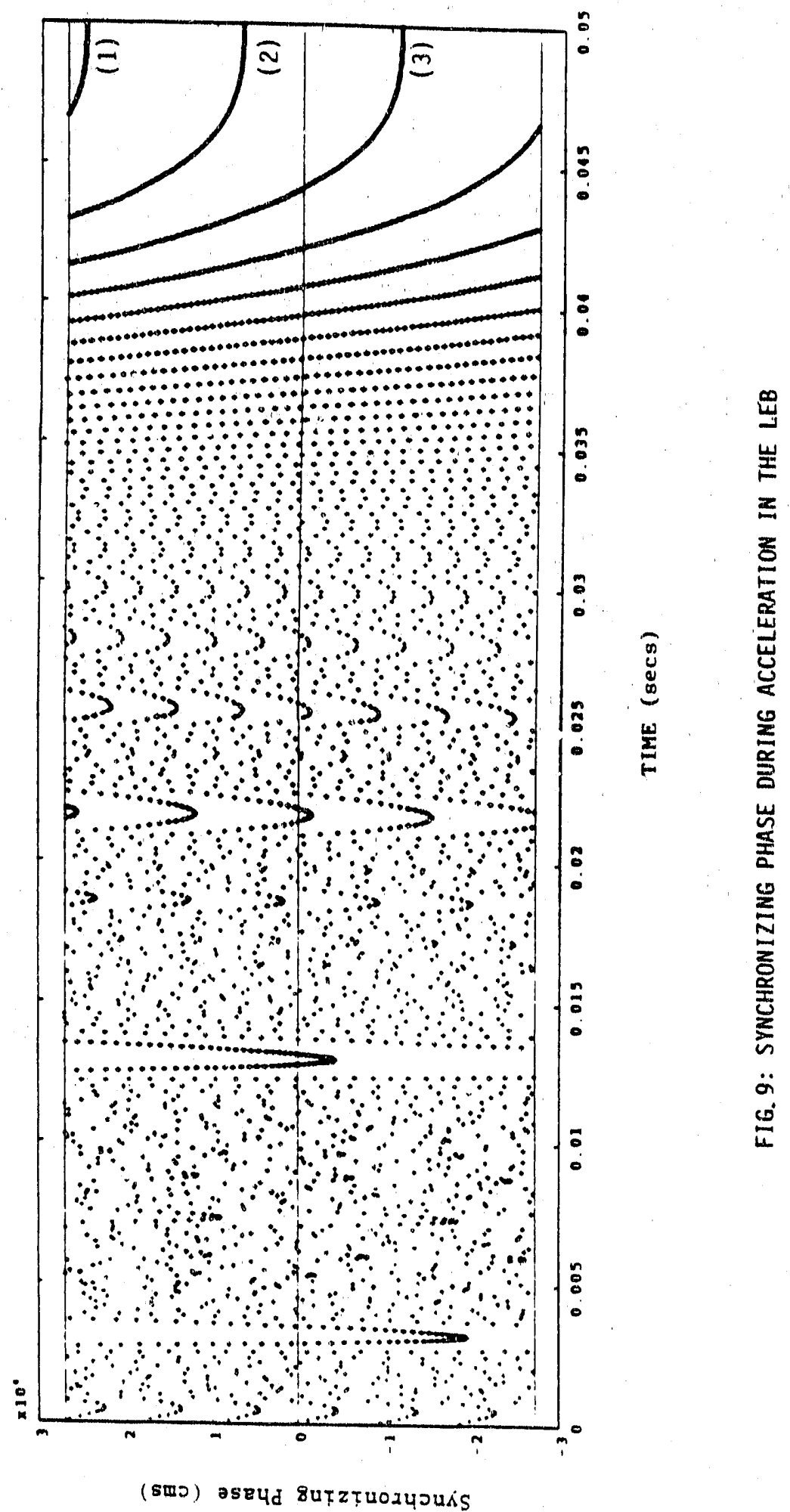


moved anywhere in the orbit if we set the initial relative beam position, $L_{M E B}(0)-L_{L E B}(0)$, appropriately. For example, to make the decay to zero we have to set $L_{M}{ }^{B B}(0)-L_{L E B}(0)$ to be equal to $68.38 \mathrm{~m}$; then curve 2 will exponentially decay to zero. Experimentally this can be achieved by controlling the injection time from the Linac into the LEB and the LEB RF turnon time. That is, by knowing the time when the first pulse in the Linac has been inj, xted, the RF switch-on time can be adjusted to a little later time so that there is enough tine to arrange the MEB phase relative to the LEB phase to a desired point in the ring. By doing this initial phase adjustment we can achieve correct phase at the instant of transfer provided we have kept control of the phase to an adequare degree of accuracy throughout the cycle. This path length, $L_{\psi}$ becomes our trip plan for the LEB beam bunch. We set up a detection scherne digitally, to enable the deviation of the synchronizing phase from the designated trip plan for every MEB turn and then use this error information to carefully adjust the LEB RF frequency such that it is forced to take up a desired value sufficiently in advance within a reasonable time before extraction. We have plans to "feed forward" by refreshing the trip plan every cycle so that a pseudo-adaptive loop is arranged to adjust itself without human interference.

The detection of phase error will fail if the B-field fluctuation is too large since it may lead to a very large phase error., If it is greater than the LEB circumference within the rettling time of the feedback controller then we will lose control over the phase error. We evaluated this case for a fractional B-field error of $10^{-3}$. It showed that the phase error exceeded one LEB circumference in 6 milliseconds. This is quite tolerable since we can design a feedback controller that adjusts the LEB RF frequency to control the phase error within 6 milliseconds. Since the B-field error is expected to be less than $4 \times 10^{-4}$ we anticipate acceptable behavior.

In Figure 10 we have sketched the block diagram of the complete digital control system. In this scheme we have shown two inputs for the 'Synchronizing Phase Error Detector.' These can be either from the beam position monitors located at the reference points or from the two RF signals. The trip plan for the LEB such as the one shown in Figure 9 is calculated in the Trip Plan Generator. Apart from the computed phase, it also includes subtle information such as (1) the synchronous phase of the LEB and MEB reference particles when the two RF signals are used as the input for the Phase Error Detector (2) transfer line delays (3) the kicker rise time and (4) the steady delay in the electronics associated with synchronization. The phase error is computed for each MEB turn on a real time basis in the detector by time-slicing and then time-tagging the arrival time of the LEB beam bunch to the reference point. This information is then subtracted appropriately with the inexorable trip plan. The error in the Synchronizing phase is then processed in the Sliding-Mode Controller block $^{10}$. The output of the Sliding-Mode Controller is the required frequency modulation which would cornpensate for the error in the synchronizing phase. The main RF frequency for normal acceleration of the beam is obtained from the B-field using high resolution $A$ D converters. Using modern high speed logic we believe that it is possible to compute the phase error and also develop the control signal within one MEB turn which is about 13.2us. Details af the processor and the controller design are more complicated and hence are not shown here. It is however described in reference 9. 


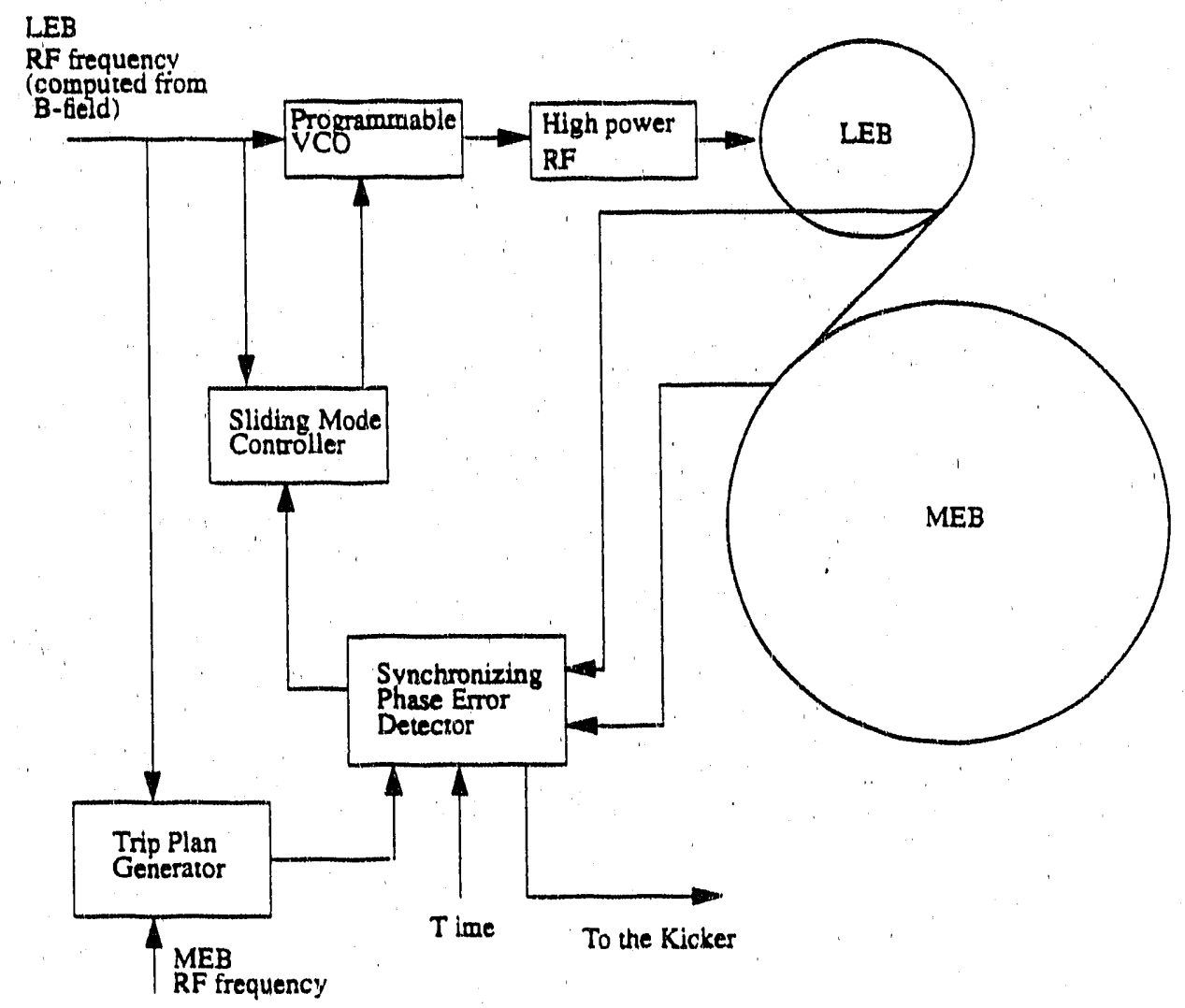

Figure 10

\section{REFERENCES}

1. F. D. Wells and S. P. Jachim, "A Technique for Improving the Accuracy and Dynamic Range of Beam Position - Detection Equipment," IEEE PAC, 89CH2669-0, Proc. 1989.

2. J. Crisp et al., "A Programmable High Power Beam Damper for the Tevatron," IEEE Trans. Nucl. Sci., Vol. NS-32, No.5, October 1985.

3. C. E. Dickey, "Irradiation of Fiber Optics in the SSC Tunnel," private communication to be published in 1990 .

4. E. Peschardt and J.P.J. Sladen, "Phase Compensated Fibre-Optic Links for the LEP RF Reference Distribution," IEEE Particle Accelerator Conference, Chicago, IL, p. 1960, 1989 
5. J. A. Dinkel et al, "Synchronous transfer of beam from the NAL fast cycling booster synchrotron to the NAL main ring system," IEEE Trans. Nucl. Sci., Vol. NS-20, No. 3 , June 1973.

6. Y. Kimura et al., "Synchi onous transfer of beam from the booster to the main ring in the KEK proton synchrotron," IEEE Trans. Nucl. Scl,, Vol. NS-24, No. 3, June 1977.

7. Site-Specific Conceptual Design of the SSC, Technical volume 1, December 20, 1989.

8. Griffin, J. E.: 'Private technical discussions, FNAL, Chicago, Illinois, February 1990.

9. Mestha, L.K.: 'Phase-control scheme for synchronous beam transfer from the Low Energy Booster to the Medium Energy Booster', SSC Laboratory Report, 1990 (To be issued).

10. K. S. Yeung, Private technical disc ussions, Department of Electrical Engineering, University of Texas at Arlington, Arlington, TX, 1990 


\section{SSC BEAM POSITION MONITORING SYSTEM}

The BPM system contains the most important instrumentation for beam control. Position pick-ups, each with four electrodes, will be located at most quadrupoles in all the accelerators. Doublet pulses of $1 \mathrm{~ns}$ duration and $16 \mathrm{~ns}$ separation are produced as the beam traverses the pickups. The peak-to-peak amplitude of pulses varies from $0.5 \mathrm{~V}$ at machine commissioning to $20 \mathrm{~V}$ at operating intensity. The detector signals will be processed and digitized in the niches, and the digitized data stored in registers for readout by the control system. At least three methods of analog signal processing are being considered to meet accelerator requirements.

Each sensing device is a detector consisting of four $15-\mathrm{cm}, 50 \mathrm{ohm}$ strip transmission lines placed above, below, and to both sides of the beam as shown in Figure 1 and Figure 2. To maximize the signal to noise ratio, each electrode subtends most of one quadrant. So that the electrodes will not be aperture defining elements, the beam tube bulges out around them. The electrodes are also recessed $2 \mathrm{~mm}$ outside the aperture so that synchrotron radiation cannot strike the $n$. The characteristic impedance of the four electrodes must be matched within $\pm 0.25 \%$ to hold the electrical to mechanical center difference to 0.005 in., and to be within $\pm 0.5 \%$ of 50 ohms to control reflections at the cable interface. The detectors are rigidly welded to the spool pieces in each half-cell for alignment purposes and therefore operate at $4 \mathrm{~K}$. The 8000 vacuum feedthroughs used in the collider must isolate beam vacuum from liquid helium. Because of their great quantity and inherent difficulty of replacement they must be very reliable (MTBF $>870 \times 10^{6} \mathrm{hrs}$ ). The feedthrough and cable to the outside of the cryostat form an integral assembly and are composed of 316 Stainless Steel (S.S.) and $\mathrm{Al}_{2} \mathrm{O}_{3}$ ceramic. The cable dielectric is $\mathrm{Si} \phi_{2}$. The 0.142 in. dia. S.S. jackets of the four cables form a part of the hermetically sealed cryostat which confines the liquid helium. The cables will hold off $20 \mathrm{~atm}$. LHe, be radiation resistant, tolerate welding and be very rugged.

Since beam detector directionality is not required in the collider, one end of each stripline is shorted. This measure saves construction cost, reduces heat leak, and improves reliability. The integral cable assemblies mate to SMA bulkhead vacuum feedthroughs which isolate guard vacuum from atmosphere. These feedthroughs are at the outside surface of the cryostat and are readily replaceable if damaged. All BPM signals will be brought to processor racks in the tunnel niches from the adjacent 3 consecutive upstream and downstream halfcells. The longest cable runs are $270 \mathrm{~m}$.

The BPM electronics must provide position and intensity signals under various operating conditions: during machine commissioning; at full intensity collider operation; in fault diagnosis; and during specialized accelerator studies. Optimal performance in a variety of applications requires front-end processing tailored to the various modes and ring lucations.

For maximum sensitivity and maximum dynamic range in sensing trains of bunches separated by $5 \mathrm{~m}$ intervals, down-converted amplitude-to-phase conversion is used ${ }^{1}$. Signal processing is done using only one harmonic of the RF bunching frequency. With $15 \mathrm{kHz}$ wide bandpass filters in the IF section and $15 \mathrm{~dB}$ noise figure limiters, resolution of $100 \mathrm{~nm}$ should be achieved. The good sensitivity of these channels at low beam current will be valuable in steering the beam through the first turn commissioning. For this purpose, it is not necessary to instrument every half-cell, and for that reason, AM/PM processing will be used at the BPM stations adjacent to the niches, every three cells. These locations use $30 \mathrm{~m}$ of $7 / 8$ 


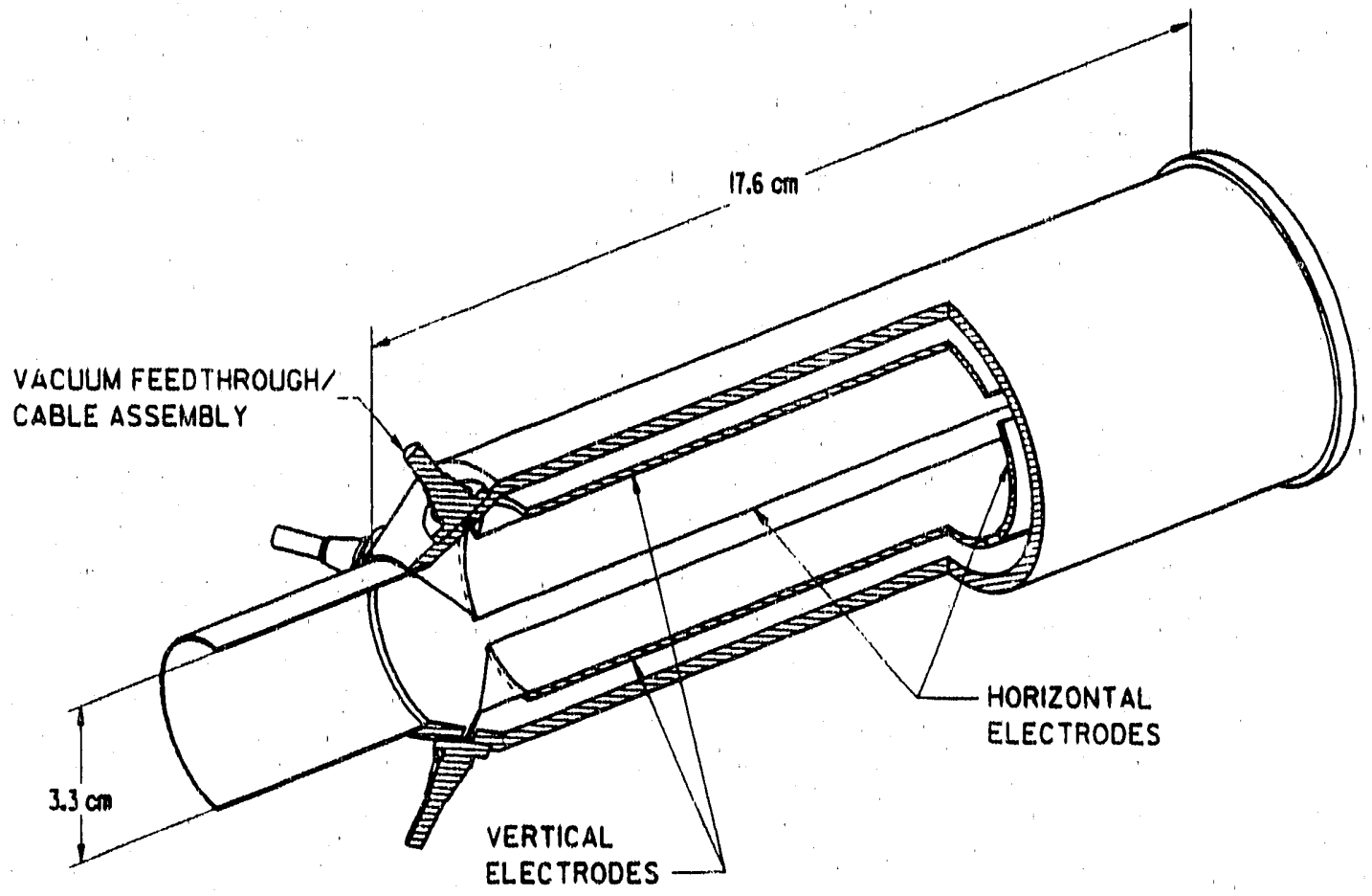

Figure 1. Isometric view of beam position monitor.

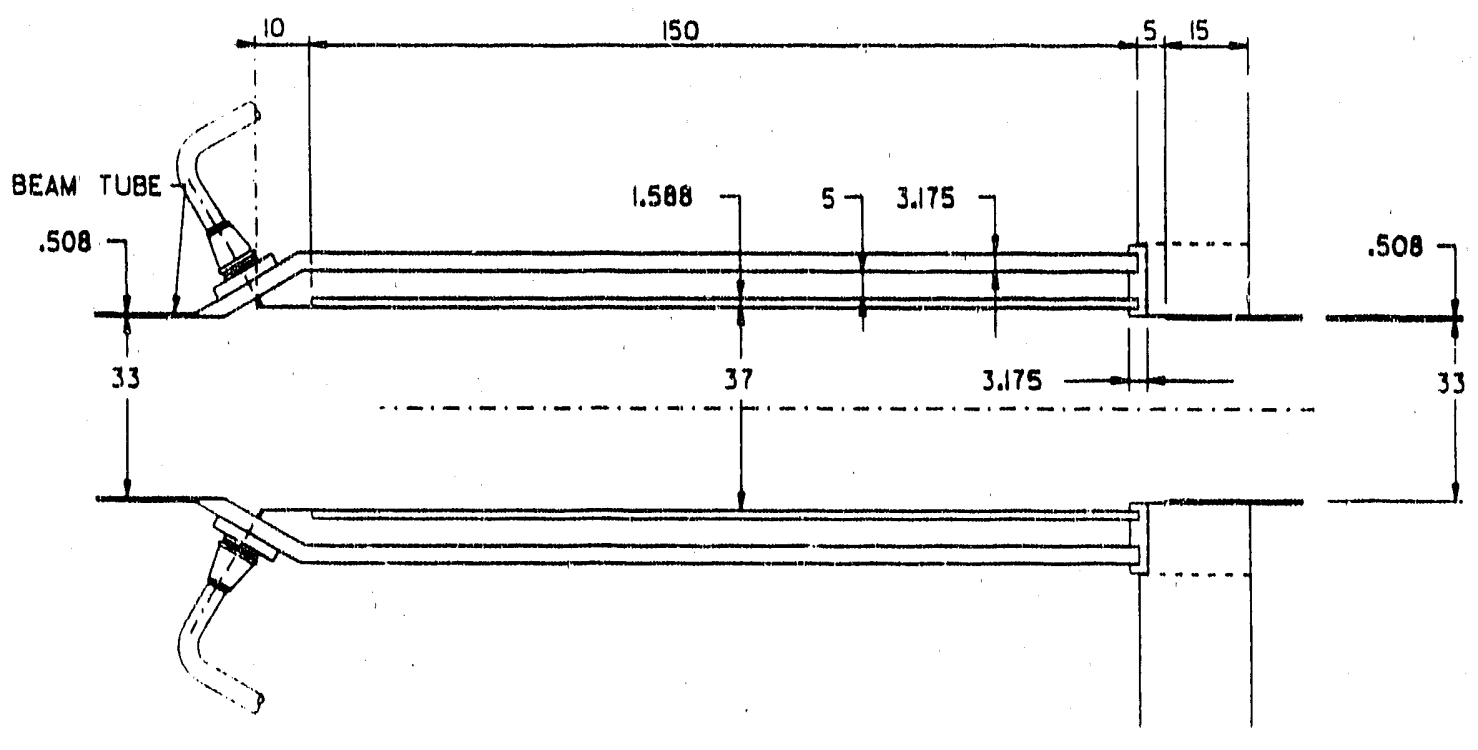

NOTE:

ALL DIMENSIONS ARE IN MILLIMETERS

Figure 2. Plan vicw of beam position monitor. 
in. solid copper jacketed corrugated cable, making the niche BPM's the most sensitive locktions in the accelerator. Position monitoring near the IR's will also use this system.

At the BPM's most distant from the niches, skin effect losses even in high quality, low loss cable remain excessive for the pulse risetime to be preserved. Therefon, located close to the pickups, will be peak detection circuits which effectively down-convert the high frequency components. The diode detectors provide the bulk of the front-end BPM processing, are the least costly of the mathods, and use lower quality RG-213 cable to transmit rectified pulses to the niches. The diode circuits have limited dynamic range, about $35 \mathrm{~dB}$, and must be hardened against radiation. To measure the transverse position of a particular bunch, adjustable timing signals are used to gate the 12-bit ADC. These timing signals are derived from the global timing system described in the next section. Each station will be equipped with First In First Out (FIFO) memories that retain the most recent ten thousand turns of data. These will be used for occasional readout, for example for post mortem af:er a beam abort to reconstruct the beam orbit. Under special conditions, all position monitors must be able to measure orbit distortions of about 10 microns peak amplitude to locate orbit cusps.

For specialized beam studies, bunch-by-bunch and turn-by-turn processing will be implemented in a few places in each accelerator. Such a system will be able to measure the position of any bunch, any sequence of bunches, the same bunch on every bearn crbit (turn), or any other definable bunch pattern. The electronics will employ 8-bit $100 \mathrm{MHz}$ flash ADD converters, digital intensity normalizing, and FIFO memories to record position histories. To obtain reasonable resolution, wideband sum and difference signals are obtained using hybrid transformers integral to each detector. The flash converters clocked at $60 \mathrm{MHz}$ talie one sample of the vertical position, horizontal position, and intensity of each bunch. The ratios of differences to sums are taken digitally and are written into fast memories, which can be read by the control system. A 100Kbyte memory could store the position data of 6 full turns, or the position of a single bunch on $10^{5}$ turns. The electronics would be similar to the front end processing done in the Tevatron beam dampers 2 .

\section{SSCL PRECISION TIMING SYSTEMS}

\section{Introduction}

The timing systems of the SSCL generate the individual timing triggers in the accelerator complex. They are used by the synchronization of beam transfer, beam dump and beam position data acquisition systems, which require a jitter of $s 100 \mathrm{psec}$. Their use by the ramping and corrector magnets require a jitter of $\leq 1 \mathrm{msec}$. Finally, the systems provide a phase compensated RF reference. It is no small task to distribute a timing signal with a jitter and precision of $\leq 100$ psec over a geographic area of approximately 200 square mules. Thie collider itself will contain 161 niches, spaced evenly 540 meters apart. Each of these niches must receive the high precision timing signal.

The precision timing system incorporates features from the Tevatron, CERN and the SLC project at SLAC. An SSCL timing system consists of a distribution network which carries RF reference signals and timing modules which count the number of cycles of the RF reference signal. At predeternuned counts, timing modules produce trigger signals. For more precise timing, individual RF cycles can be subdivided. 
There are 7 different timing systems: the Linac, LEB, MEB, HEB, SSC Top, and SSC Bottom Beam Syncs, and Global Timing. T:e Beam Sync systems are distributed to their respective accelerators, while the Global Timing is distributed to all accelerators. The systems perform the same functions but have different master clocks. The master clock of a system is the origin of the RF reference signal of a system. For the Global Timing, the master clock is phaselocked to the $1,000,000$ th harmonic $60 \mathrm{~Hz}$ line frequency, or $60 \mathrm{MHz}$. For the Beam Syncs, the master clock is phaselocked to the RF in the accelerator cavities.

During acceleration, the Beam Sync systeins are approximately $60 \mathrm{MHz}$, but do sweep a significant frequency range (47 to $60 \mathrm{MHz}$ for the LEB). Other than the effects of the FM signal, the systems may be considered identical in function and construction. Wo will confine our observations to the Global Timing System (GTS).

\section{Goals of the GTS}

We require that timing modules trigger simultaneously to \pm 100 psec anywhere in the accelerator complex. Jitter and repeatability of individual modules should be better than 100 psec. A high mean time between replacement (MTBR) is required, as the GTS must be uperating properly for the collider to operate. MTBR should exceed 100,000 hours for the individual modules. Self diagnostics should allow faults to be determined during production and as an aid to troubleshooting during installation and operation. If possible, the system should give indications of how soon various failures are predicted for modules.

\section{Network Topology}

The precision, cost and reliability of the timing : ystem is strongly influenced by how the signals are actually routed - the distribution network topology. The intention of the distribution network topology is to minimize jitter, uncertainty, drift and cost, while maximizing reliability.

The simplest distribution network, a horseshoe (Figure 3a) would have onc fiber strung from niche to niche, with a repeater at each niche. This would minimize cost of fiber used to $\$ 262,500$, but would result in a probable increase in jitter, uncertainty and drift of 22 times that of a single repeater, and a worst case increase of 484 times that of a single repeatex. The loss of a single repeater would cripple all the down stream timing circuits, or as much as $1 / 2$ of the collider.

The opposite extreme, as shown in Figure 3b ( star net), would be to string a separate fiber to each niche, which would require bundles of as many as 484 fibers to be strung in the cable conduit. It would minimize the jitter, uncertainty ard drift to that of one repeater, but would require $\$ 63,381,000$ of fiber. Loss of any repeater w ould affect only one niche.

A compromise star-star network (Figure 3c) would distribute one fiber to each sector, which would then subdistribute to each niche. This results in bundles of no more than 13 fibers. The total cost of fiber is $\$ 982,200$. The probab \& jitter, uncertainty and drift are 1.41 times that of a single repeater, and the worst case is 2 times that of a single repeater. Loss of a subdistribution repeater would affect only one $n$ che, while loss of a distribution repeater would affect at most one sector. The star-star netwo $\mathrm{rk}$ will require 10 more repeaters than the other systems. The system is shown in more detail in Figure 4. 


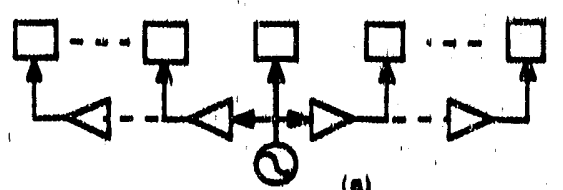

(a)

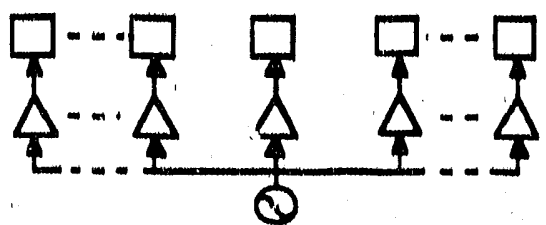

(b)
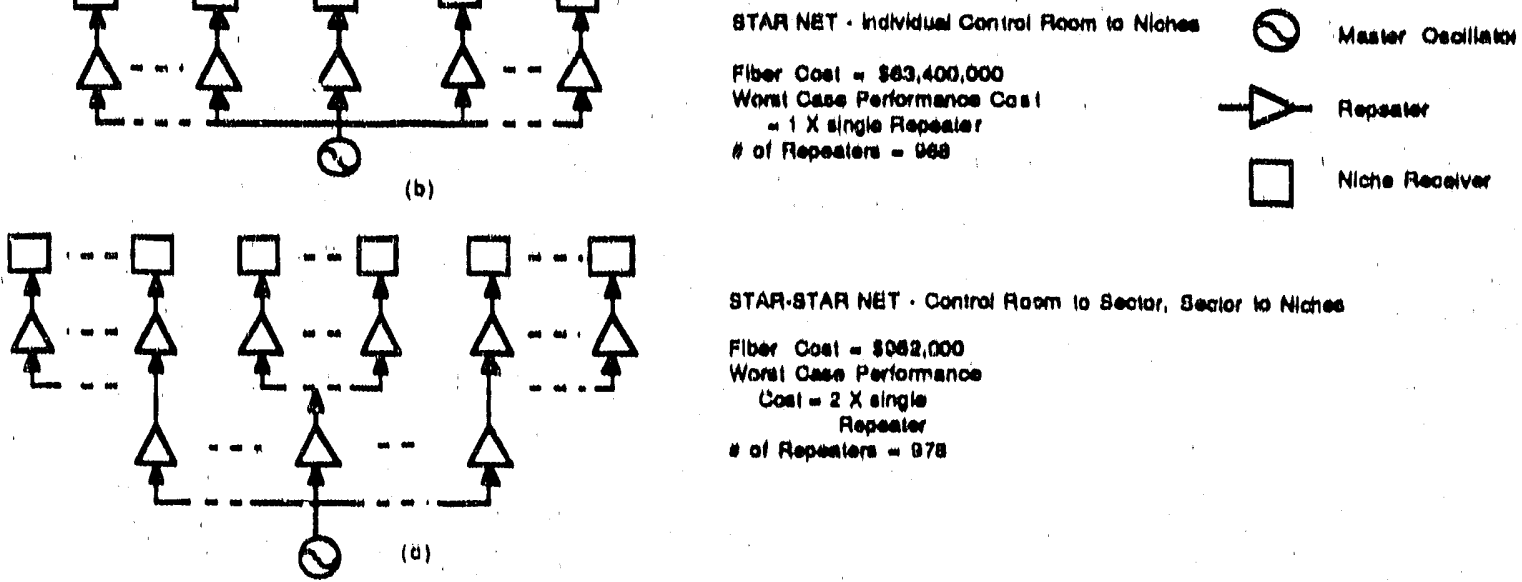

gTAP.STAR NET - Control Room lo scolor, Bector to Nichece

Flber Cont - soan,000

Worel cana Purtormanos

Coal - 2 X oingle

- of Acopentere on

FIG, 3: TIMING DISTRIBUTION NETWORK

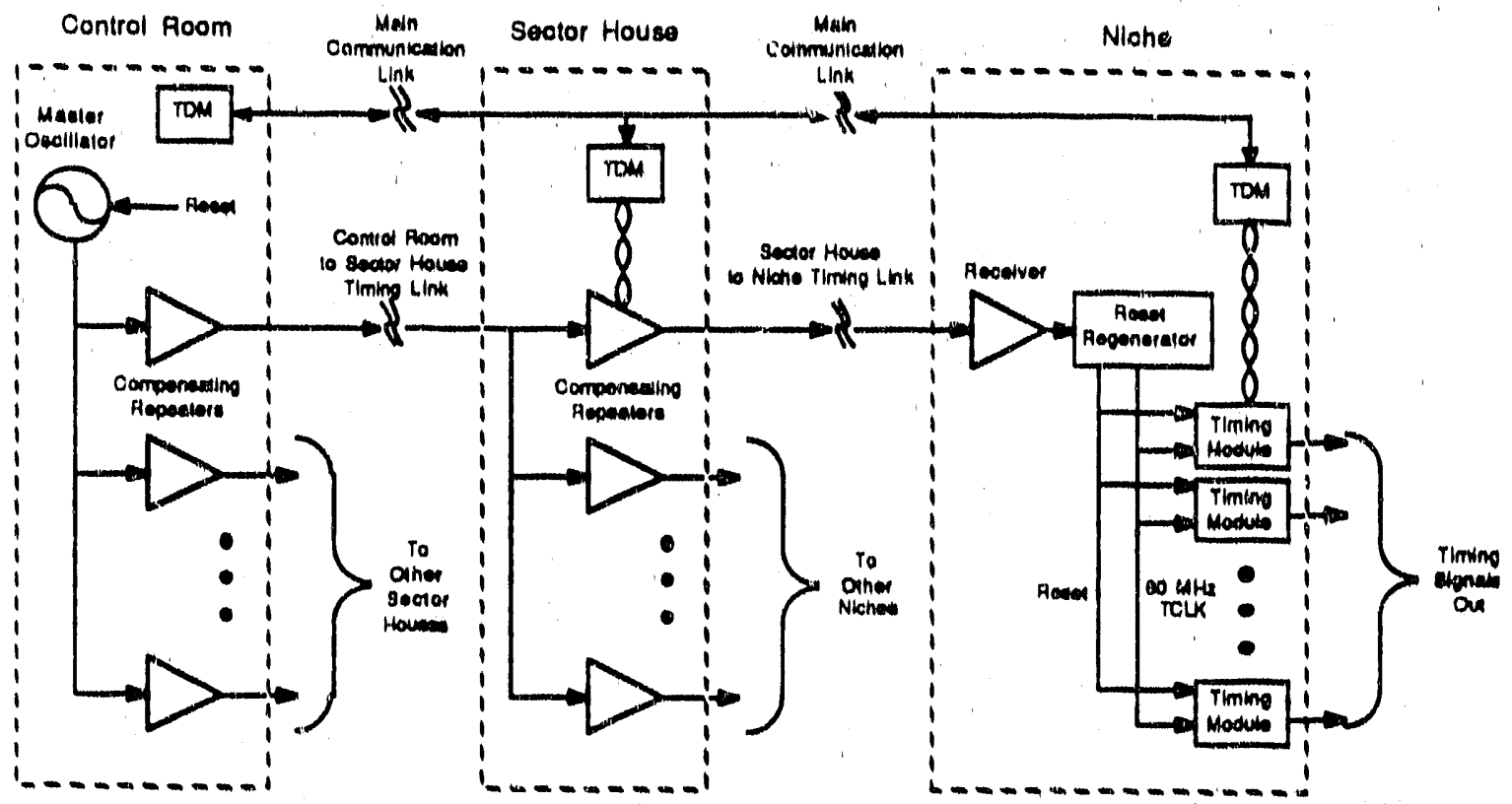

FIg. 4: TIRING SYSTEM BLOCK DIAGAAM

Installation of the network is no small task. There is some question of the long term radiation resistance of the fiber, as silica becomes more opaque as it is exposed to neutrons. The fibers are shielded underneath a minimum of 18 inches of rubble and concrete. The niches are 540 meters apart, and contain the pull boxes for the fiber. To reduce the number of 
splices required, the fiber bundle is pulled from one niche and respooled at the next. This process is repeated, with appropriate breakouts being made, until the entire network is strung.

\section{High Accuracy Timing Signal Transmission}

Either coaxial or fiber optic cables could be used to distribute the timing signal. Fiber optios are superior to coax in their cost, attenuation, variation of propagation velocity with temperature and common mode noise rejection characteristics. The radiation resistance of fiber optics is comparable to coax ${ }^{3}$. Fiber optic links have also been used in the Tevatron and LEP timing systems.

Vartations in temperature of $\pm 5^{\circ} \mathrm{C}$ will make variations of as much as $\pm 13 \mathrm{nsec}$ in triggering time from one side of the collider to the other due to the variation in propagation velocity with temperature. A technique originally developed by Feschardt and Sladen ${ }^{4}$ and enhanced for this application allows the temperature variation to be compensated (Figure 5). It does this by measuring the phase shift through the fiber round trip and compensating for any variation.

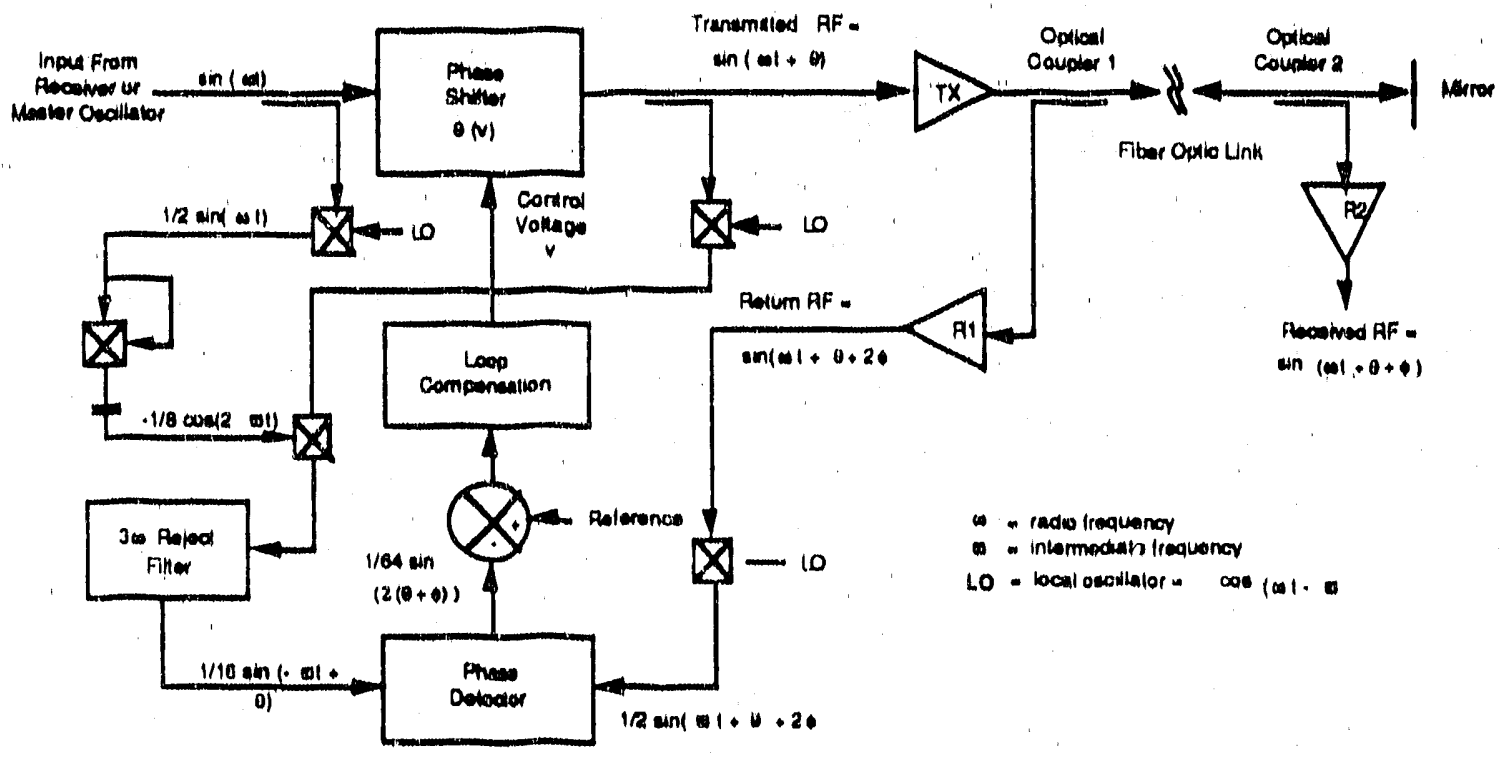

FIg. 5: PHASE.COMPENSATED FIBER OPTIC LINK (Adapled from Pescherdf and Sladen)

As seen in Fig. 5, an input signal, designated "From Receiver/Signal Processor", is assumed to be the phase reference $V_{i}$

$$
V_{i}(t)=\sin (\omega t)
$$

This is passed through the phase shifter, and results in $V_{t}$

$$
V_{1}(t)=\sin (\omega t+\theta(v))
$$


where the phase shift $\theta$ is a function of the control voltage $v, V_{t}$ is then transmitted over a single mode optical fiber. The media adds an additional phase shift of $\phi=\omega \tau$, where $\tau$ is the one way time delay of the media. Optical Coupler 2 plisks off a fraction of the signal, which is converted into $V_{\text {reo }}$ by Recelver $R X 2$.

$$
V_{\text {roo }}(t)=\sin (\omega t+\theta(v)+\phi)
$$

The signal which is not absorbed by RX2 is reflected back to the transmitter, where it is picked off by Optical Coupler 1 and converted into $V_{\text {ret. }}$

$$
V_{\text {ret }}(t)=\sin (\omega t+\theta(v)+2 \phi)
$$

$V_{1}$ is down converted by a local osciliator LO. The down converted frequency is represented by $\omega$. The frequency of $L O$ is set so $\omega$ is about $100 \mathrm{KHz}$.

$$
\begin{gathered}
V_{L O}(t)=\cos ((\omega-\varpi) t) \\
\left.V_{i}(t) V_{\text {LO }}(t)\right|_{\text {LowPass }}=\left.\sin (\omega t) \cos ((\omega-\varpi) t)\right|_{\text {LowPass }}=\frac{1}{2} \sin (\varpi t)
\end{gathered}
$$

This down converted reference is then multiplied by itself and the DC component removed, resulting in $-\frac{1}{8} \cos (2 \omega t)$. This is multiplied by a down converted image of $V_{t}$ and the 3rd harmonic removed:

$$
-\left.\frac{1}{8} \cos (2 \omega t) \sin (\Phi t+\theta(v))\right|_{\varpi \text { BandPass }}=\frac{1}{16} \sin \left(-\omega_{i}+t_{(v)}\right)
$$

$V_{\text {ret }}$ is also down converted, resulting in

$$
\begin{aligned}
\left.V_{\text {ret }}(t) V_{\text {LO }}(t)\right|_{\text {LowPass }} & =\left.\sin (\omega t+\theta(v)+2 \phi) \cos ((\omega-\sigma) t)\right|_{\text {LowPass }} \\
& =\frac{1}{2} \sin (\omega t+\theta(v)+2 \phi)
\end{aligned}
$$

When these last two signals are multiplied together in the phase detector and harmonics removed, the result is $\mathrm{V}_{\mathrm{PD}}$ :

$$
\left.V_{P D}(t)=\frac{1}{64} \cos (2 \theta(v)+2 \phi)\right)
$$

If the error amplifier and the loop filter feedback to the phase shifter, they will cause

$$
V_{P D}(t)=\frac{1}{64} \cos (2(\theta(v)+\phi))=\text { constcist }=V_{r e f}
$$

or

$$
\theta(v)+\phi=\text { constant }
$$


provided that $\theta(v)$ is approximately linear and has a positive slope, and the loop filter stabilizes the loop. But this last equation is merely the condition that the phase shift from the input to the receiver output be constant. Therefore any changes in $\phi$ with time, temperature, etc, will result in a change in $\theta(v)$ which cancels it out.

At CERN, this resulted in a reduction of the variation of the delay of the LEP RF reference line, the longest of which is $7.9 \mathrm{Km}$, from \pm 8 nsec with the feedback loop off to \pm 19 psec with the feedback loop on, over a $20^{\circ} \mathrm{C}$ temperature range. Extrapolation to the requirements of the SSC show that \pm 100 psec stability should bi: achievable.

Two additional modifications are required for the SSC. "The system must be modified when used as a Beam Sync for the lower energy boosters. This is I sause of the wide variation in operation frequency of these accelerators. Since the phase compensated line keeps the total phase shift through a line constant, the net time delay of the $\mathrm{sy}$ stem is a function of frequency:

$$
\tau=(\theta(v)+\phi) / \omega=\text { constant } / \omega
$$

This may be handled by using a TDC which keeps track of the total transit time. This number is then used to control a variable delay line, rather than a variable phase shifter. Under this control scheme, the system keeps the time delay, rather than the phase, constant (Figure 6). An aliernative may be to account for the tirne delay variation with frequency in the database of the central control computer, and redistribute new trigger times as required. The hardware solution is perhaps better, since it requires about the same level of complexity and would greatly reduce the amount of communication required betwcen the timing modules and the central computer.

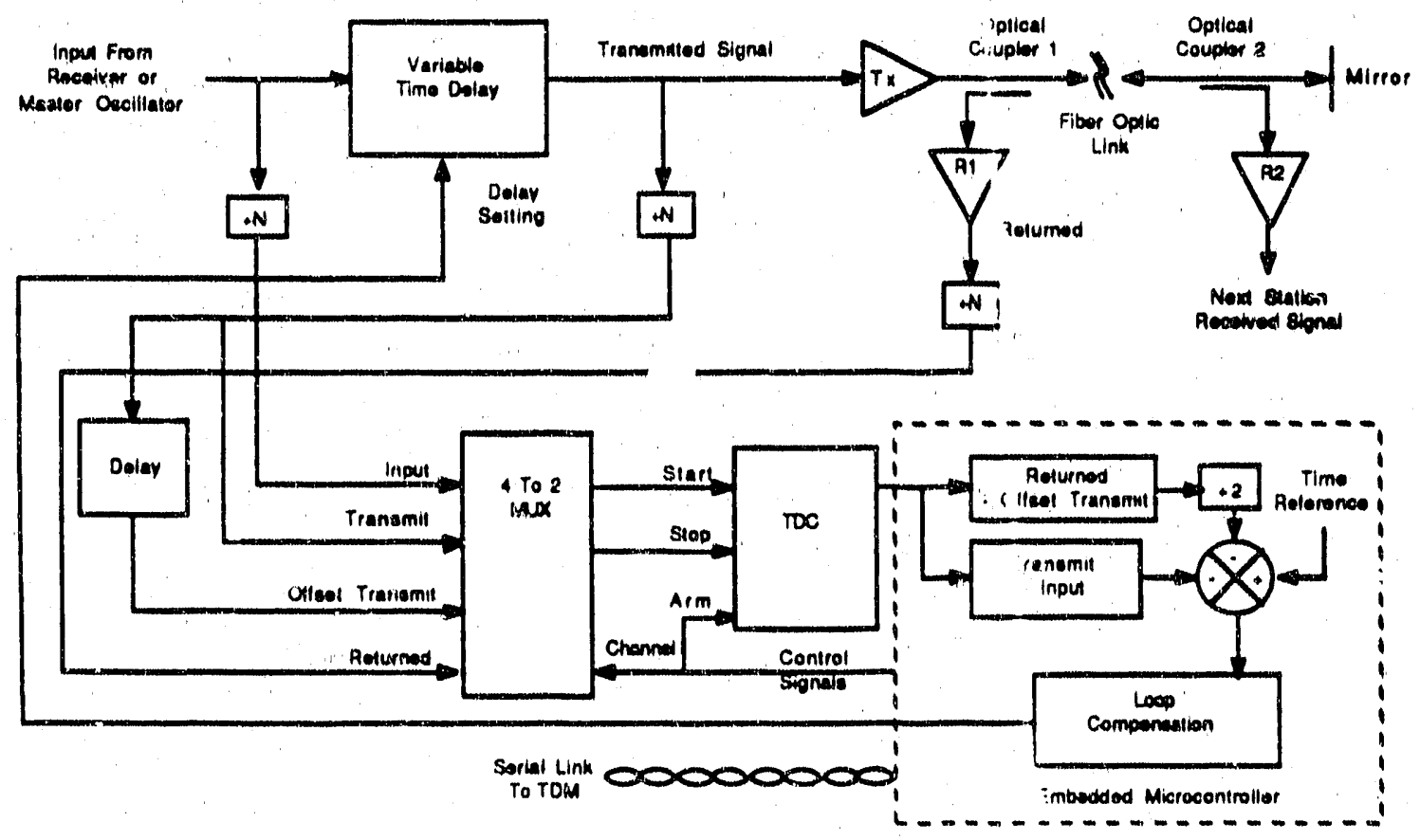

FIg. 6: TIME-COMPENSATED FIBER OPTIC INK 
A reset mechanism must also be provided which allows the timing modules to be set back to a zero count (Figure 4). This is done by removing a pulse from the transmitted pulse train. The missing pulse is detected and generates a reset signal. The reset signal is used by timing modules to reset their internal counters to zero. The RF timing frequency itself is regenerated using a phase locked loop (PLL) in a process similar to that used in high speed serial transmissions (Figure 7). This regeneration process greatly enhances the phase stability and noise margin of the signal. The signal is then distributed locally to the niche or recombined with the reset signal for subdistribution.

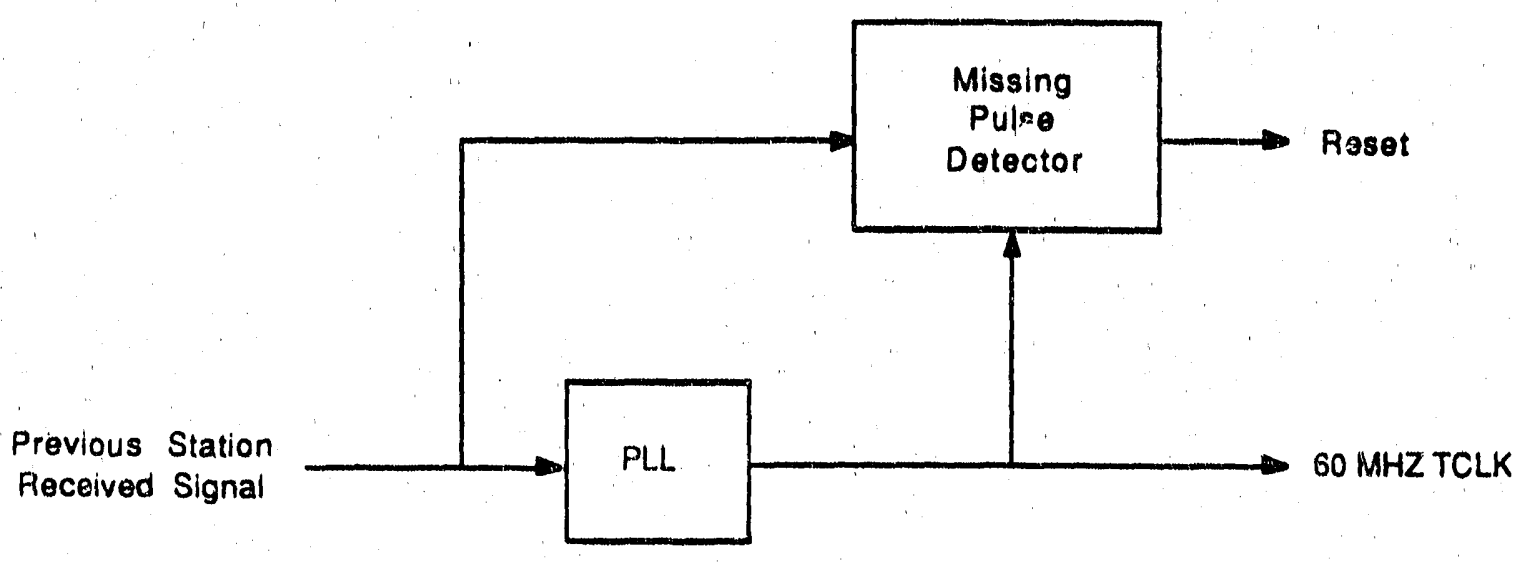

Fig. 7: RESET REGENERATOR

A diagnostic signal is returned from the loop filter to the central computer. This diagnostic measures the required compensation and hence is a direct measurement of the phase shift of the line. Each repeater's nominal phase shift can be recorded in a database in the central computer and periodically compared with the current value. A large shift in compensation would indicate that some component in the system is shifting drastically and will need to be replaced at the next maintenance cycle.

\section{Timing Module Operation}

The timing modules themselves are elementary (See Figure 8). The number of requested time clock cycles required is down-loaded from the central computer to an individual timing module via a dedicated serial link to a Time Domain Multiplexer/Demultiplexer. This is received and stored by a dedicated microcontroller unit (MCU). The MCU holds the least significant bits of the requested time word (RTW) on the input of an equality detector.

The RF reference signal TCLK enters the High Speed Counter, which simpiy tallies the number of clock cycles since the counter was last reset, or since overflow. The size of the High Speed Counter is considerably smaller than the RTW, but gives the MCU enough time to anticipate the instant when the RTW is equai to the total number of cycles since reset . When the number of cycles counted by the High Speed Counter is equal to the least significant bits of the RTW, a pulse is generated which alerts the High Speed Arming Circuit, which in turn alerts the MCU. The MCU then compares the rest of the RTW with the number 


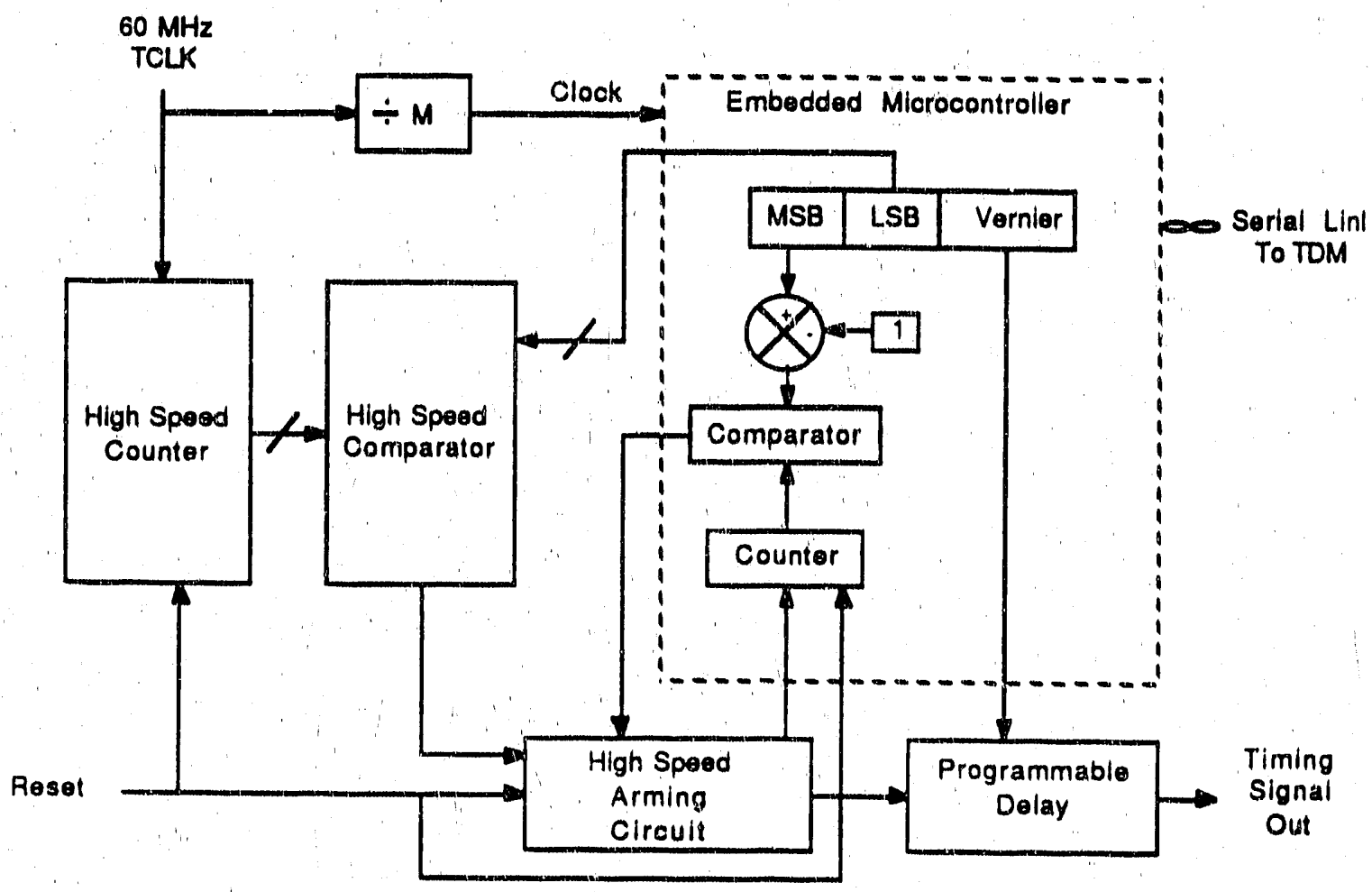

Fig. 8: TIMING MODULE

of alerts received from the High Speed Arming Circuit. Once the rest of the RTW minus one matches with number of alerts, the High Speed Arming circuit is armed by the MCU. When the next equality is detected, the High Speed Arming Circuit produces an output pulse. This pulse is delayed by a fraction of a cycle by a programmable delay line, which acts as a vernier on the RTW.

The MCU clock is slaved to TCLK, assuring that its software counters are synchronized to TCLK at least to the accuracy required to properiy anticipate and arm the High Speed Arming circuit. More than one timing module can be serviced by one MCU, which allows a reduction in cost and complexity, especially in the serial communication link to the central computer. For timing applications which do not require very high accuracy, the vernier delay line can be left out. For applications which require only crude timing, most of the high speed circuitry may be omitted.

\section{Timing System Calibration}

The central computer provides RTW's which are compensated for delay time from niche to niche, FM effects in the lower energy boosters, etc. Each of the timing systems must be calibrated and the calibration stored in the central database. For example, the difference in phase between two clocks separated by a communication link of time delay $\tau$ is given by 


$$
\begin{aligned}
\Delta \phi & =\int_{t_{0}}^{t_{0}+\tau} \omega(t) d t \\
& \equiv \tau \omega\left(t_{0}\right)
\end{aligned}
$$

It is clear that the time delay from the master oscillator to each niche must be known with high accuracy. This calibration procedure is done in two steps. The first, a crude calibration, allows the SSCL, to be commissioned but does not provide the ultimate level of accuracy of the system to be achieved. The technique is simply to replace the master oscillator with a high stability atomic clock, and then to synchronize a second atomic clock to the temporary master. This atomic clock is portable and connected to a timing module. Once this is done, the second atomic clock and its attendant timing module may be moved quickly from niche to niche and the mobile timing module compared with the count achieved by the the timing modules at each niche.

The accuracy of this system is limited by the maximum speed with which a precision atomic clock may be moved from point to point in the system and by the phase stability of state-of-the-art atomic clocks. The best accuracy requires a hydrogen maser clock. The speed of a clock through the collider is limited to about $15 \mathrm{Km} /$ hour. An overland route might speed this up considerably, but might unduly vibrate the atomic clock.

The second calibration step can proceed once protons can circulate through the collider. The protons travel at a known velocity, for a known distance, and their time of flight from niche to niche can be determined with arbitrary accuracy and precision. By using the timing modules to note the exact time of arrival of a single bunch of protons, the time delays of the system may be calibrated out to an accuracy of better than $100 \mathrm{psec}$ at all locations in the accelerator complex.

\section{SSCL BEAM TRANSFER SYNCHRONIZATION}

\section{Introduction}

At present there are two schemes for achieving synchronous transfer of bunched beams from one circular accelerator to another in various laboratories around the world 5.6 . In the Booster to Main Ring transfer at Fermilab, a phase-locking scheme is in use. In this approach, at a suitable time before injection to the Main ring, the phase and frequencies of the Booster RF are locked to the Main Ring RF system. When the RF phase error is zero synchronous transfer is triggered. In the corresponding transfer at KEK in Japan the phase matching is done by using a phase-slippage scheme. In this scheme, at a predetermined time before the transfer, the Booster RF frequency is offset by $10 \mathrm{Khz}$ relative to the Main Ring RF frequency. As a result, since the Main Ring RF frequency is constant, the RF phase of the Boos er ring slips relative to the Main Ring. Phase coincidence is detected and then a kicker system is fired to transfer the beam. Some emittance dilution occurs owing to the mismatch of bunch spacing; that is, an inherent drawback of the system.

The phase-locking scheme gives good results when there is sufficient time, first to achieve the RF lock, and then to perform the "cogging" operation in which the bunch pattern is rotated into its correct orientation. In the SSC, especially for the beam transfer between the 
Low Energy Booster (LEB) and the Medium Energy Booster (MEB), this scheme is $r$ it suitable because at top energy the LEB is operating very close to transition which makes the cogging time too large 7,8 and the peak B-field does not have a long flat top since it is derived from the biased sinusoidal resonant power source. (It has been judged impractical to derive a long flat top by switching an auxiliary power supply). However, for all other transfers at SSC this scheme can be applied since the B-field can be held constant until the transfer process is completed.

The ideal scheme for synchronizing beam transfer from the LEB to MEB is to keep control of the relative beam phase throughout the acceleration. Since the frequency of the LEB is modulated by about $20 \%$ during acceleration, the concept of relative phase must be generalized and for that purpose we introduce a new term "synchronizing phase" for a phase that is controlled through the cycle and acquires its standard meaning when the two frequencies become equal. In this method, transfer is triggered at the instant the synchronizing phase vanishes. This avoids emittance dilution due to mismatch of bunch spacing as in the phase-slippage scheme, even with large bunch separation (e.g., 90m instead of the nominal $5 \mathrm{~m}$ ). The scheme has two essential elements. First, it involves the accurate detection of the synchronizing phase error by comparing the measured values with the known "trip plan" stored in the compute. The trip plan and the synchronizing pha.ie are related terms with the trip plan being ideal values of the synchronizing phase at discrete intervals through the cycle. Second, the measured phase error is passed through a feedback controller digitally, so that a compensating RF frequency shift is generated to minimize the error. In this way the synchronizing phase is controlled to a pre-programmed trajectory. In the following section we discuss the method qualitatively and show how it will be possiole to detect the synchronizing phase at regular intervals. The feedback controller is not expiained. A detailed analysis of the scheme is contained in reference 9 .

\section{Principle of the Phase-Control Scheme}

To begin with let us assume that there is no frenuency error in the $L$ IB and MEB RF systems. Transfer line delays and measurement errol in beam position (du: for example, to coupled bunch oscillations) are ignored. Also, to understand the basic principle, let us assume that the synchronous phase of the reference particle in the beam bunch is constant throughout the acceleration cycle. With these assumptions in mind v'e can write an expression for the path length covered by the beam for a time duration of $\tau$ as follows.

$$
L_{L E B}(\tau)=\frac{2 \pi R_{L}}{h_{L}} \int_{0}^{t} f_{L}(t) d t+L_{L E B}(0)
$$

$$
\text { where, } \begin{array}{ll}
\tau & =\text { Time interval } \\
R_{L} & =\text { Radius of the orbit } \\
h_{L} & =\text { Harmonic number for the LEB } \\
f_{L}(t) & =\text { RF frequency of the LEB }
\end{array}
$$

(Numerically $\mathrm{L}_{\mathrm{LEB}}$ is roughly $10^{7} \mathrm{~m}$ at transfer time. Since a longitud: aal uncertainty of less than $10.2 \mathrm{~m}$ is required at that time, it is clear that absolute control of a parameters to the corresponding precision of one part in $10^{9}$ is an impractical way of synchronizing transfer). 
Taking advantage of the constancy of the MEB frequency, the path length in the MEB can be writter:

$$
\mathrm{L}_{\mathrm{MEB}}(\tau)=\frac{2 \pi \mathrm{R}_{\mathrm{M}}}{\mathrm{h}_{\mathrm{M}} \tau+\mathrm{L}_{\mathrm{MEB}}(0)}
$$

The difference in path lengths, $L_{\psi}=L_{M E B}-L_{L E B}$, is equal to the synchronizing phase. The phase when defined in this form has units of length. To solve for $L_{\psi}$, the following equation is used for the LEB RF frequency with usual notations.

$$
f_{L}(t)=\frac{h_{L} c}{2 \pi R_{L} \sqrt{1+\left(\frac{M_{p} c^{2} / e}{\rho 0 B(t)}\right)^{2}}}
$$

Where $\rho$ is equal to the effective bending radius. The accelerator guide field $B(t)$ varies with time according to the following expression:

$$
B(t)=B_{\min }+\frac{B_{\operatorname{mix}}-B_{\min }}{2}(1-\cos \omega t)
$$

Using Eq. 15 in Eq. 13 the path length, $L_{L E B}$, is calculated numerically with a fine time step of 1 ns. We assumed $L_{L E B}(0)=0=L_{M E B}(0)$, i.e., the beam bunch in the $L E B$ was assumed to have started at the same time as the MEB beam bunch from the reference points. The reference points were chosen at the beam pick up points nearest to the beginning and the end of the transfer line. For synchronous transter we would like the synchronizing phase to be equal to zero. Since we are dealing with a circular machine, when the MEB beam bunch has completed one single turn, the LEB beam bunch will have compieted a few whole turns plus a semi-turn. The semi-turn is due to the difference in the two RF frequencies throughnut acceleration and the fact that the circumference ratio between the MEB and the LEB is ..ot a whole integer. Hence $\mathrm{L} \psi$ can be rewritten in the following form.

$$
L_{w}=L_{M E B}-L_{L E B}=\left(N_{\text {MEB }}+\gamma_{M}\right) 2 \pi R_{M}-\left(N_{L B B}+\gamma_{L}\right) 2 \pi R_{L}
$$

Where $N_{M E B}, \gamma_{M}$ and $N_{M E B}, \gamma_{L}$, represents the whole and the semi-turn completed by the MEB and the LEB reference beam bunch respectively. The whole turn is of no significance for synchronous transfer for obvious reasons. Hence we simply drop it and consider only the fractional part representing the incomplete turm.

For synctironization, at the time of transfer the fractional part $\gamma_{M}$ and $\gamma_{L}$, must be equal to zero. In Figure $9, L_{\psi}$ is plotted each time the MEB beam bunch retums to the reference point (i,e, when $\gamma_{M}$ becomes zero). It can be seen clearly from this figure that after about $47 \mathrm{~ms}$ the LEB beam bunch tends to come back to a fixed point in the orbit more frequently than before. We see three curves that are approaching constancy. (Three curves are due to the fraction " $1 / 3$ " in the MEB to LEB circumference ratio: when the ratio is a whole integer, then we would have only one curve.) The decay is due to the fact that the difference between the two RF frequencies is narrowing as the time approaches the nominal $50 \mathrm{msec}$ transfer time at which $B(t)$ is maximum.. These curves settle down eventually to a fixed value; for example $68.38 \mathrm{~m}$ is the final settling value for curve 2 . The final settling value can be 

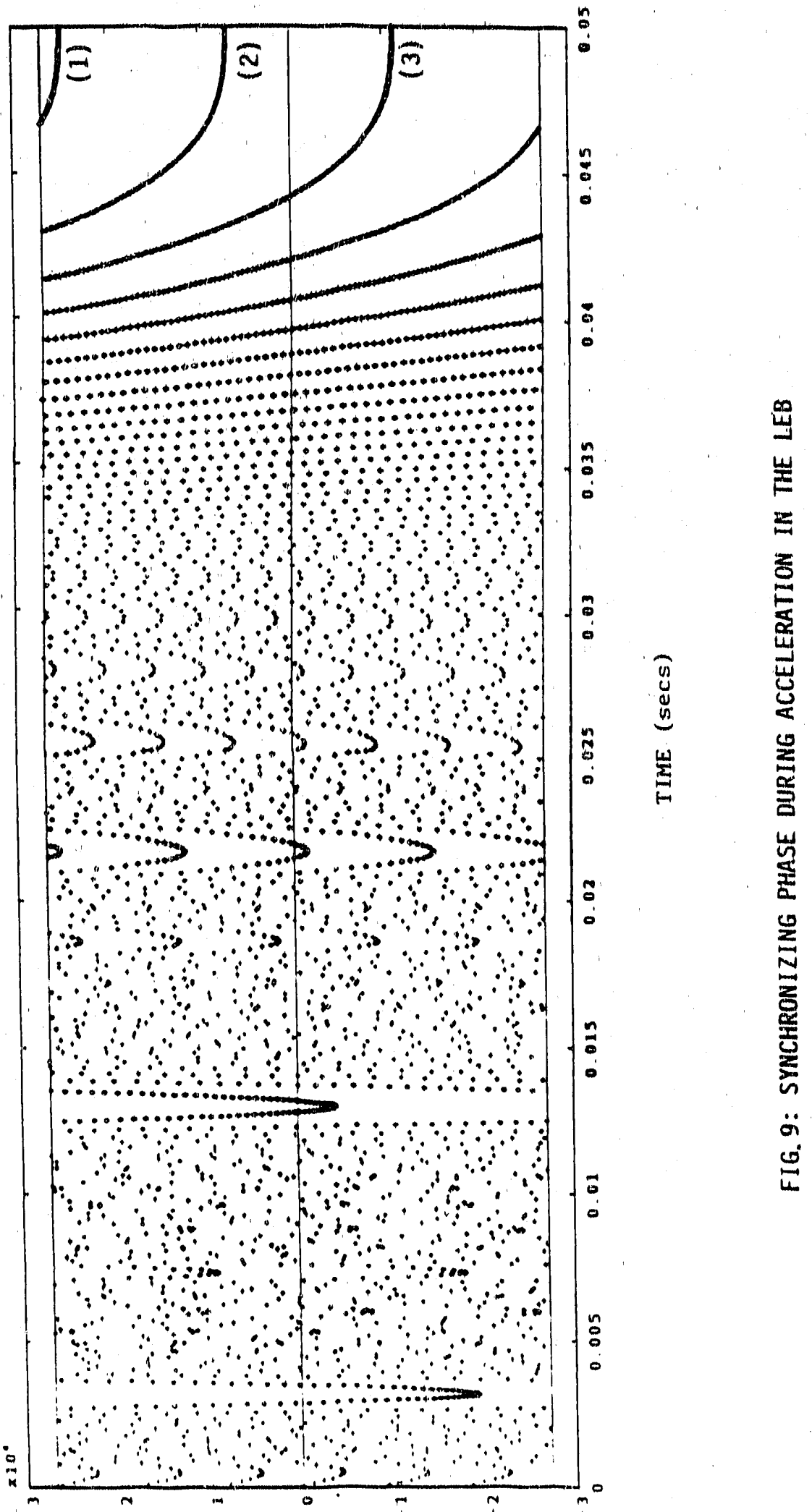

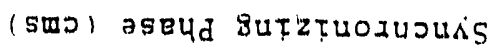


moved anywhere in the orbit if we set the initial relative beam position, $L_{M E B}(0)-L_{L E B}(0)$, appropriately. For example, to make the decay to zero we have to set $L_{M E B}(0)-L_{L E B}(0)$ to be equal to $68.38 \mathrm{~m}$; then curve 2 will exponentially decay to zero. Experimentally this can be achieved by controlling the injection time from the Linac into the LEB and the LEB RF turnon time. That is, by knowing the time when the first pulse in the Linac has been injected, the RF switch-on time can be adjusted to a litele later time so that there is enough time to arrange the MEB phase relative to the LEB phase to a desired point in the ring. By doing this initial phase adjustment we can achieve correct phase at the instant of transfer provided we have kept control of the phase to an adequate degree of accuracy throughout the cycle. This path length, $L_{\psi}$ becomes our trip plan for the LEB beam bunch. We set up a detection scheme digitally, to enable the deviation of the synchronizing phase from the designated trip plan for every MEB tum and then use this error information to carefully adjust the LEB RF frequency such that it is forced to take up a desired value sufficiently in advance within a reasonable time before extraction. We have plans to "feed forward" by refreshing the trip plan every cycle so that a pseudo-adaptive loop is arranged to adjust itself without human interferenco.

The detection of phase error will fail if the B-field fluctuation is too large since it may lead to a very large phase error. If it is greater than the LEB circumference within the settling time of the feedback controller then we will lose control over the phase error. We evaluated this case for a fractional $B$-field error of $10^{-3}$. It showed that the phase error exceeded one LEB circumference in 6 milliseconds. This is quite tolerable since we can design a feedback controller that adjusts the LEB RF frequency to control the phase error within 6 milliseconds. Since the B-field error is expected to be less than $4 \times 10^{-4}$ we anticipate acceptable behavior.

In Figure 10 we have sketched the block diagram of the complete digital control system. In this scheme we have shown two inputs for the 'Synchronizing Phase Error Detector.' These can be cither from the beam position monitors located at the reference points or from the two RF signals. The trip plan for the LEB such as the one shown in Figure 9 is calculated in the Trip Plan Generator. Apart from the computed phase, it also includes subtle information such as (1) the synchronous phase of the LEB and MEB reference particles when the two RF signals are used as the input for the Phase Error Detector (2) transfer line delays (3) the kicker rise time and (4) the steady delay in the electronics associated with synchronization. The phase error is computed for each MEB turn on a real time basis in the detector by time-slicing and then time-tagging the arrival time of the LEB beam bunch to the reference point. This information is then subtracted appropriately with the inexorable trip plan. The error in the Synchronizing phase is then processed in the Sliding-Mode Controller block ${ }^{10}$. The output of the Sliding-Mode Controller is the required frequency modulation which would compensate for the error in the synchronizing phase. The main RF frequency for normal acceleration of the beam is obtained from the B-ffeld using high resolution $A / D$ converters. Using modern high speed logic we believe that it is possible to compute the phase error and also develop the control signal within one MEB turn which is about 13.2us. Details of the processor and the controller design are more complicated and hence are not shown here. It is however described in reference 9. 


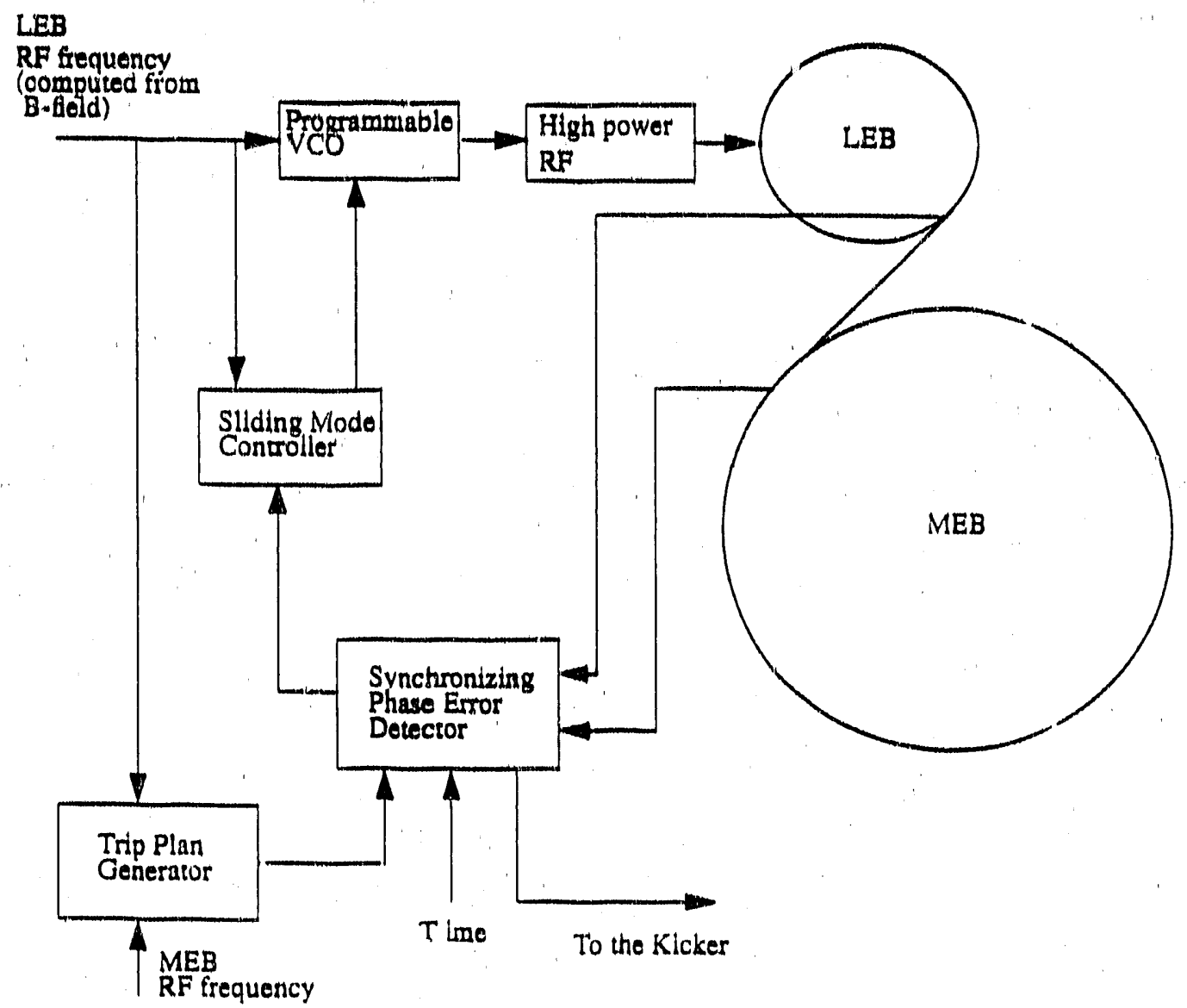

Figure 10

\section{REFERENCES}

1. F. D. Wells and S. P. Jachim, "A Technique for Improving the Accuracy and D vnamic Range of Beam Position - Detection Equipment," IEEE PAC, 89CH:669-0, Proc. 1989.

2. J. Crisp et al., "A Programmable High Power Beam Damper for the Tevatron,' IEEE Trans. Nucl. Sci., Vol. NS-32, No.5, October 1985.

3. C. E. Dickey, "Irradiation of Fiber Optics in the SSC Tunnel," private communica ion to be published in 1990.

4. E. Peschardt and J.P.J. Sladen, "Phase Compensated Fibre-Optic Links for the LI P RF Reference Distribution," IEEE Particle Accelerator Conference, Chicago, IL, p. 960, 1989 
5. J. A. Dinkel et al. "Synchronous transfer of beam from the N.AL fast cycling booster synchrotron to the NAL main ring system," IEEE Trans. Nucl. Scl., Vol. NS 20, No. 3, June 1973.

6. Y. Kimura et al." "Synchronous transfer of beam from the booster to the main ring in the KEK proton synchrotron," IEEE Trans. Nucl. Sci., Vol. NS-24, No. 3, June 1977.

7. Site-Specific Conceptual Design of the SSC, Technical volume 1, December 20, 1989.

8. Griffin, J. E.: 'Private technical discussions, FNAL, Chicago, Illinois, February 1990.

9. Mestha, L.K.: 'Phase-control scheme for synchronous bearn transfer from the Low Energy Booster to the Medium Energy Booster', SSC Laboratory Report, 1990 (To be issued).

10. K, S. Yeung, Private technical discussions, Department of Electrical Engineering, University of Texas at Arlington, Arlington, TX, 1990 

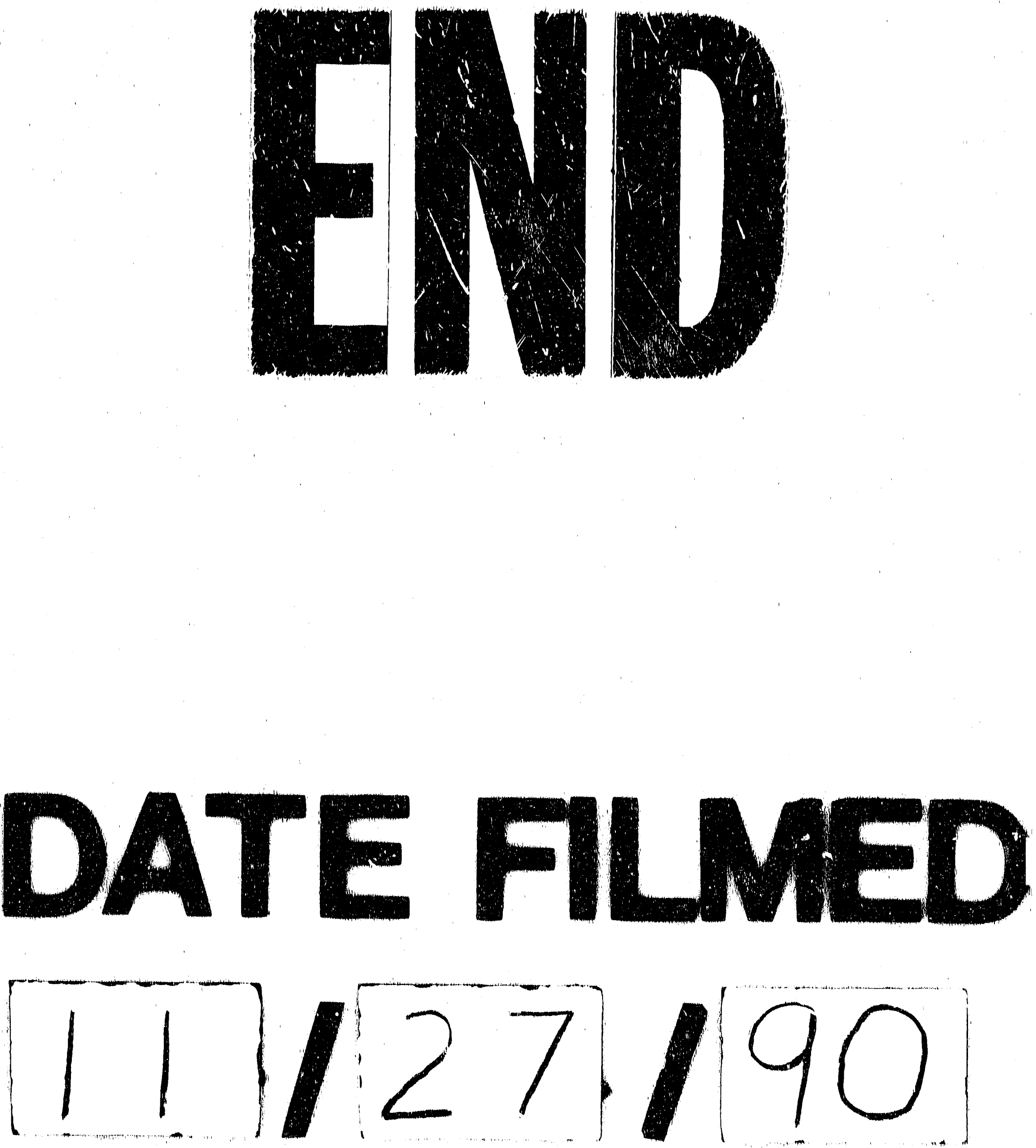
$\Upsilon$ 